FPS-Vidicon Television Cameras for Ultrafast-Scan Data Acquisition

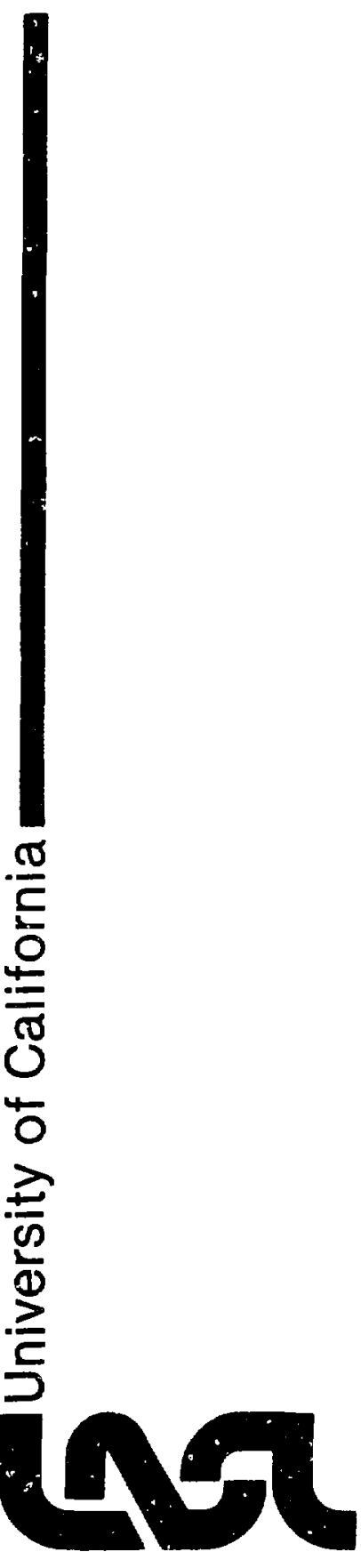




\title{
FPS-VIDICON TELEVISION CAMERAS FOR ULTRAFAST-SCAN DATA ACQUISITION
}

by

Bruce W. Noel and George J. Yates

\begin{abstract}
Two ultrafast-scan ( $<8 \mathrm{~ms} /$ field) television (TV) camera prototypes have been developed for closed-circuit data-acquisition applications. The line and field rates are quasi-continuously adjustable. The number of lines, the integration duty cycle, and the scan direction are a mong the other adjustable parameters. Typical resolution at the fastest scan rate is $>500 \mathrm{TV}$ lines per picture height with a corresponding dynamic range (to light input) of more than 100. The cameras use the unique properties of FPS vidicons and specially designed electronics to achieve their performance levels and versatility.

The advantages and disadvantages of FPS vidicons and of antimony trisulfide and silicon target materials in such applications are discussed in detail.

All of the electronics circuits are discussed. The sweep generator designs are treated at length because they are the key to the cameras' versatility. Emphasis is placed on remotely controllable analog and digital sweep generators. The latter is a complete CAMAC-compatible subsystem containing a 16-function master arithmetic logic unit.

Pulsed and $\mathrm{cw}$ methods of obtaining transfer characteristics are described and compared. The effects of generation rates, tube types, and target types on the resolution, determined from contrast-transfer-function curves, are discussed.

Several applications are described, including neutron TV pinhole, TREAT, and barium-release experiments.
\end{abstract}

\section{INTRODUCTION}

Television (TV) cameras specifically designed for closed-circuit data acquisition may differ in many respects from those used by the broadcasting industry. Several examples follow. The automatic light-level controls found in broadcasting cameras are not wanted in data-acquisition cameras, whose vides output voltage must be calibrated relative to the input light level. The ability to trade resolution for dynamic range, ${ }^{*}$ or vice versa, may be necessary. Further, broadcasting cameras often require only a relatively small dynamic range (say, 30 or 50 to 1 ), whereas data-acquisition cameras may require a very large dynamic range (1000 or more to 1 ). A need for single-field or single-frame capabilities will put stringent requirements on image lag or image

*We define the dynamic range as the difference between the peak video output and rms random-noise voltages. 
buildup ("soak") characteristics of the cemera tube. Field durations that differ from the standard may be important in some applications.

We have designed, built, and field-tested several prototypic versions of two models of TV cameras specifically intended for data-acquisition applications requiring ultrafast scan $(<8 \mathrm{~ms} /$ field). Both models have single-field and single-frame capabilities and offer short scan-line and field durations while still providing good or superior resolution. The line and, field durations are variable over a wide range and can be selected in several ways. We have operated the cameras with line durations of 8 $\mu \mathrm{s}$ to $80 \mu \mathrm{s}$ and field durations of $2.800 \mathrm{~ms}$ to 16.384 ms. ${ }^{*}$ To use the available scan time efficiently, the retrace interval per line is maintained at $5 \%$ of the line duration for even the shortest lines. These performance specifications are attained by using a mixed-field (electrostatic deflection and magnetic focus) image tube known as the FPS (focus projection and scanning within the same tube element) vidicon. ${ }^{1-4}$ We refer to the two prototypes as Model-1 and -2 FPS cameras.

Some other features of the cameras include wide dynamic range, high peak-îo-peak (p-p) video output voltage (useful in high-noise environments) into long $50-\Omega$ cables, and remotely resettable sync and sweep generators for slaving to other cameras or for restarting the scans following a trigger pulse initiated by, or otherwise time-related to, the event(s) to be viewed. The tradenff between dynamic range and resolution is facilitated by using one of two different preamplifiers, either of which can be trimmed easily for virtually any desired combination of dynamic range and resolution.

\section{DESIGN CONSIDERATIONS}

It is useful to define a figure-of-merit (FOM) for evaluating a camera's performance when it is operating in a variable-scan-rate, variableresolution mode. We define one such FOM as

$\mathrm{F}_{\mathrm{e}}=\mathrm{R}_{\boldsymbol{v}} \eta / \mathrm{N} \tau$

where $\mathrm{N}$ is the number of lines per field, $\tau$ is the line duration, $\eta$ is the ratio of active horizontal sweep interval to total line time including retrace, and $R_{v}$ is

"A circuit has been designed to lower the minimum line duration to $5.12 \mu \mathrm{s}$. the vertical resolution, all in some convenient units. The quantity $\eta$ is a measure of the relative possible information content per line that we call the image efficiency. Clearly, larger FOM velues indicate higher performance. This FOM is valid when the system bandwidth is such that the horizontal resolution equals (hence the "e" subscript) the vertical resolution. Otherwise, the FOM is

$F_{u}=R_{v} A R_{h} \eta / N r$,

where $A$ is the aspect ratio and $R_{n}$ is the horizontal resolution. The quantity $V_{\tau}$ is the fiald time exclusive of vertical retrace and vertical interval, which we assume, for ease of comparison, to be constant for any camera.*

The figure of merit, whether $F_{e}$ or $F_{u}$, is insufficient in itself to completely specify the camera parameters, but it aids in comparing potential designs, especially in choosing the vidicon tube.

\section{A. The FPS Vidicon in Ultrafast-Scan Applica- tions}

We chose to design the camera prototypes around the FPS vidicon because of its potentially high FOM, arising from two advantages it has over conventional vidicons: (1) higher possible scan rates for a given resolution and (2) higher resolution at any given scan rate.** Most conventional vidicons are magnetically deflected and focused. They can provide satisfactory focusing and hence good resolution under ultrafast-scan conditions, but then they require unreasonable high-frequency capability from the deflection circuits to make $\eta$ large. On the other hand, electrostatically deflected and focused tubes can provide satisfactory $\eta$ values because highfrequency deflection is easy, but with unacceptably low resolution because of relatively poor focusing. The FPS vidicon uses electrostatic deflection and magnetic focus superimposed in the same volume to achieve both fast deflection capability and good focusing. The electrostatic deflection, which is done by an internal derlectron ${ }^{5}$ assembly, permits us to

\footnotetext{
* In olir cameras, the vertical retrace and vertical interval are maintained as constant fractions of the field time and are therefore shorter than they are in standard commercial cameras. This reflects to our disadvantage when the respective FOMs are compared, but makes the comparison simpler.

** It is quite possible for a non-FPS tube to have one of these advantages and not the other.
} 
achieve very high scan rates. In our applications, electrostatic deflection has another important advantage. The available magnetic-deflection cameras that offer variable sweep rates (all of them known to us operate only at commercial or slower rates) provide only a relatively small number of rates, all of which are a multiple of a fundamental harmonic. This restriction is imposed by limitations on the deflection-coil design. No such restriction occurs with electrostatic-deflection tubes. Using analog sweep generators, we can select any desired line duration and any desired integer number of lines within the sweep and resolution limits of the tube and its amplifiers. With digital sync generators and digital control of the line duration and number of lines, we are restricted to incremental rather than continuous values, but these are much more closely spaced than they are for magnetic-deflection systems. For example, a high-quality magneticdeflection camera might be restricted to horizontal frequencies of 15,30 , and $45 \mathrm{kHz}$, with corresponding discrete line times of only $66.7,33.3$, and $22.2 \mu \mathrm{s}$. Our shortest tested line time is $8 \mu \mathrm{s}$. The line times can be varied in increments as small as $50 \mathrm{~ns}$ (limited only by the clock frequency of the present digital sync generator), and are thus quasicontinuously variable. A final disadvantage of magnetic-deflection cameras is that it is difficult to maintair constant raster size for higher-frequency magnetic swceps because the flyback pulses required from the deflection amplifiers are larger than can be handled by available transistors (that we know of).

The main focusing of the FPS tube is done by an external solenoidal focus coil. An additional electrostatic prefocus lens demagnifies the beam before it enters the magnetic field, so it is smaller in diameter for a given beam current than is a standard vidicon beam. Because of the smaller spot size, more beam diameters can be fitted along any given scan line at the target. Since the resolution of any vidicon tends to be beam-spot-size-limited rather than target-grain-size-limited, the FPS vidicon gives higher resolution than do standard magnetically focused vidicons at any given scan rate. In addition to improving the resolution, the higher electron density in the smaller beam for a given current permits its scan velocity across the target to be increased to as much as three times conventionai values while still removing all of the charge from a given site on the target. ${ }^{\circ}$ In fact, when the scan velocity is in- creased, the current can increase over its value for the same charge at lower velocity becsuse the charge removed per unit time, $\Delta Q / \Delta t$, increases.

\section{B. Target Materials}

We used a vidicon with a silicon-diode-matrix target in the Model-1 FPS camera. For that camera's intended applications, the silicon target offered several advantages over the more common antimony trisulfide $\left(\mathrm{Sb}_{2} \mathrm{~S}_{9}\right)$ targets. The major advantage was the superior "soak time ${ }^{118,7}$ of silicon targets. Soak time is what we call the time required for image buildup after a step function (or a pulse whose duration is much longer than its risetime) of input radiant flux is applied to the target. Data scanned during this time contain amplitude errors* because not all of the charge has accumulated at the read surface of the target. As shown in Fig. 1, the soak time for $\mathrm{Sb}_{2} \mathrm{~S}_{3}$ targets varies considerably from tube to tube. The data were taken using a fast-scan extended-dynamic-range (EDR) vidicon camera ${ }^{7}$ based on a commercial camera.** The scanned image was illuminated by a short $(\approx 1-\mu \mathrm{s}$ FWHM) pulse from a GenRad model 1538-A Strobotac that was operated at a 2 - to $5-\mathrm{kHz}$ repetition rate. Even the fastest tubes require $1-2 \mathrm{~ms}$ to reach $90 \%$ of final value, many tubes require more than $5 \mathrm{~ms}$, and some take more than $8 \mathrm{~ms}$. This phenomenon thus occupies from a significant fraction of to more than the total field time in ultrafast-scan applications, thereby making it difficult or impossible to arrange the experimental geometry so that important data are not scanned while the target is still soaking. Soak can also limit dynamic range in single-field readout applications because the signal amplitude is small if the soak time is comparable with the field time. Soak-time effects and the related after-image, or lag, effects are important in those applications in which there are large radiant-flux-level differences at any given target pixel from one field to the next. Examples of such situations are rapidly moving images and sinfle-field applications. Silicon-target tubes exhibit regligible soak time.

Another advantage of silicon-target tubes in some applications is their higher absolute spectral responsivity, as shown in Fig. 2 , over the entire visible

\footnotetext{
"If not extreme, these errors can be accounted for in the data analysis if the soak curves (signal out vs time with a step-function input) are known.

*-VC-20 Series, Dage/MTI, Inc.
} 


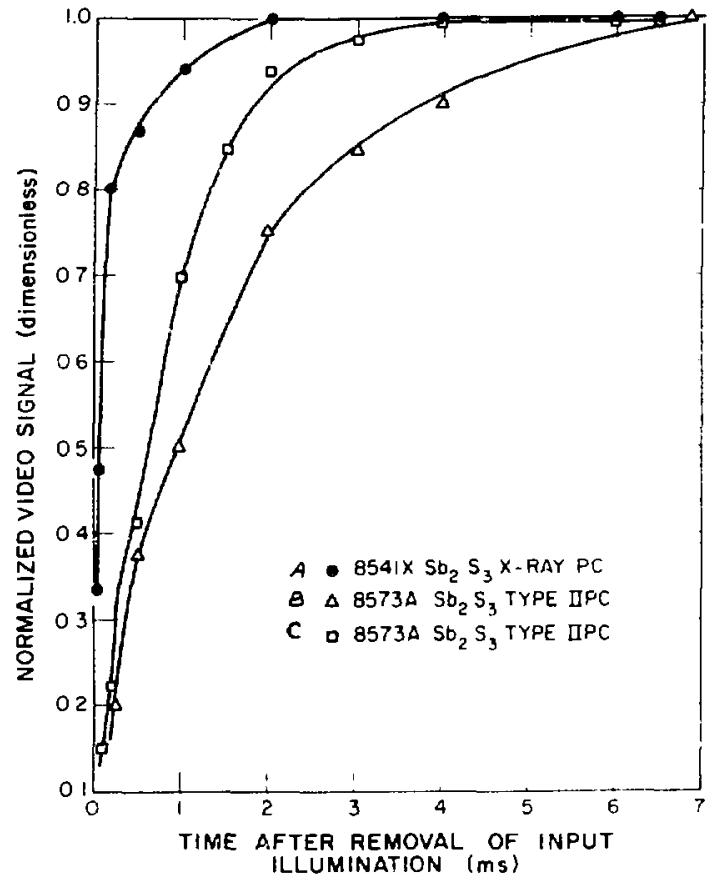

Fig. 1 .

Normalized soak curves for non-FPS $\mathrm{Sb}_{2} \mathrm{~S}_{3}$ target tubes. The data for curves $B$ and $C$ were taken with two different type 8573A tubes with standard type II photoconductive targets. The data for curve $A$ were taken with an $8541 X$ tube with an $x$-ray photoconductive target. The camera parameters were optimized individually for each tube.

wavelength band and in the near-infrared to the room-temperature silicon band edge at $1.1 \mu \mathrm{m}$.

Finally, silicon-target dark currents are typically 7 $n A$, vs typically $200 \mathrm{nA}$ for $\mathrm{Sb}_{2} \mathrm{~S}_{3}$ targets operated with large target voltages. The smaller dark current extends the dynamic range of the tube (but see further discussion below) at the bottom end of the transfer characteristic curve by reducing the fraction of the total beam current that is wasted in canceling the dark current.

The silicon-target tube also has disadvantages. $\mathrm{Sb}_{2} \mathrm{~S}_{3}$ targets are composed of microscopic grains that are much smaller than any practical beam diameter; hence the resolution is not grain-size limited. With silicon targets, some small but not always negligible resolution reduction occurs because the target surface is composed of discrete diodes that do not appear quasi-continuous when

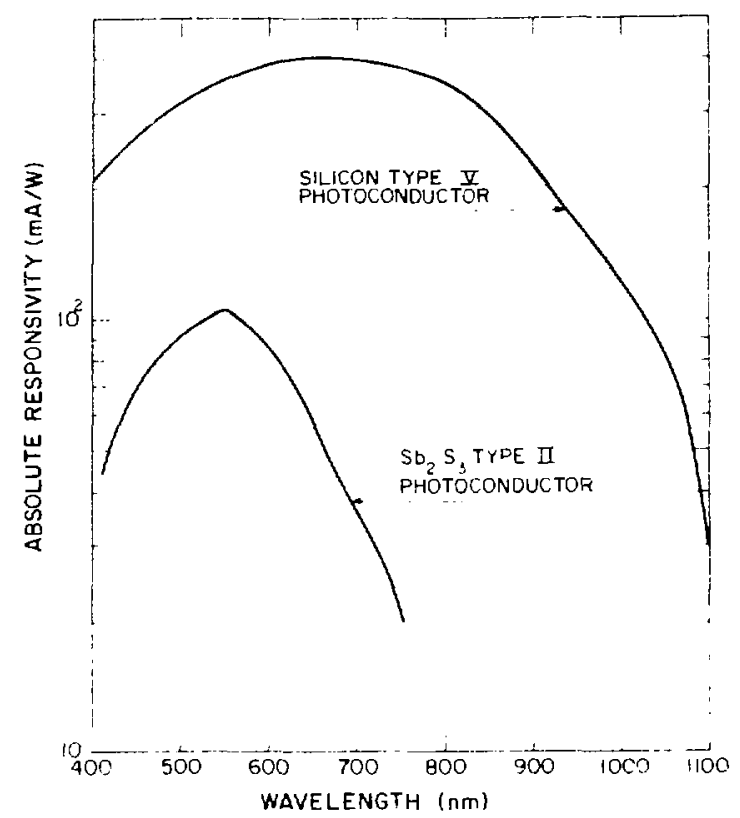

Fig. 2.

Absolute responsivities of type II $\mathrm{Sb}_{2} \mathrm{~S}_{\mathrm{s}}$-target and silicon-diode-matrix-target non-FPS vidicons. The curves were redrawn with permission from RCA $8573 A$ and RCA 4532A data sheets.

compared with the beam diameter. For example, for a 1 -inch FPS vidicon, the manufacturer specifies a limiting $(5 \%)$ resolution of 1600 lines for $\mathrm{Sb}_{2} \mathrm{~S}_{\mathrm{B}}$ targets and 1000 lines for silicon targets. Even the lower of these two figures was far more than we expected from our camera prototypes, so this disadvantage was negligible. Another disadvantage shows up when a silicon target is saturated with light. If the light is sufficiently intense, the video signal amplitude actually decreases, while at the same time a form of blooming occurs. Fortunately, one can often avoid oversaturating silicon targets, if it is a potential problem, by using sufficient care in the experimental setup.

One characteristic of silicon targets can be an advantage or a disadvantage, depending on the application. A $\log \cdot \log$ plot of video output voltage vs input low-frequencyy light is called a transfer characteristic. The slope of the transfer characteristic is known as gamma. The transfer charenteristic of silicon-target tubes has unity gamma over most of the dynamic range, while $\mathrm{Sb}_{2} \mathrm{~S}_{8}$ targets have an average gamma of about 0.7 (nearly 
unity at low levels and 0.5 or less at high levels). Silicon tubes can have greater-than-unity gamma at high light levels. ${ }^{a}$ The linear transfer characteristic of silicon-target tubes is an advantage when unity slope is required, but it can actually provide a smaller dynamic range for the silicon-target tube in terms of the range of light intensities such a tube can cover. This is mitigated somewhat by the smaller dark current of silicon targets. A limitation on the dynamic range also exits at high light levels because the peak signal current from silicon targets is about $700 \mathrm{nA}$, whereas $\mathrm{Sb}_{2} \mathrm{~S}_{3}$ targets produce up to $5 \mu \mathrm{A}$. The 700-nA value is the saturation current of those discrete diodes that are covered by the beam at a given instant. Larger currents can be achieved, but at some loss in resolution, by defocusing the read beam and thereby covering. a larger number of diodes.

One of our Model-2 FPS cameras used a tube with an " $\mathrm{x}$-ray version" of an $\mathrm{Sb}_{2} \mathrm{~S}_{3}$ target. The soak time for this tube (see Fig. 1) is negligibly small. The usefulness of such tulies as soft $\mathrm{x}$-ray detectors presumably comes about because the target material has fewer charge-carrier traps per unit volume and therefore has a lower absorption coefficient at short wavelengths. This lower trap concentration increases the carrier mobility and thus decreases the soak time and the lag. It may also cause the higher signal currents shown in Fig. 3, where the video output amplitudes of standard (type II) and $\mathrm{x}$-ray photoconductive $\mathrm{Sb}_{2} \mathrm{~S}_{3}$ targets are compared.

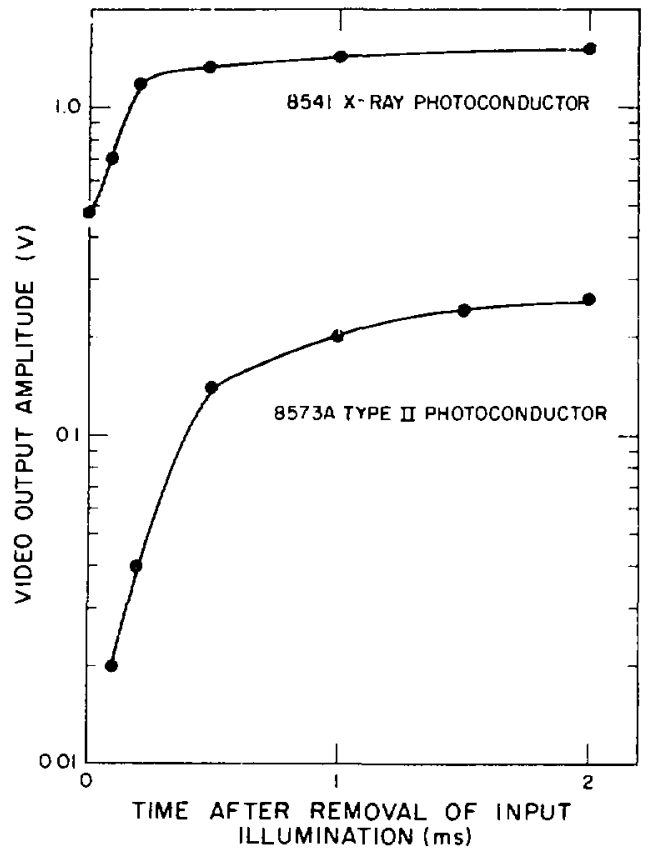

Fig. 3.

Unnormalized video ouiput amplitudes for $S b_{2} \mathrm{~S}_{3}$-target and $x$-ray photoconductive-target tubes. The data correspond to curves $A$ and $B$ in Fig. 1

Table I lists some of the FPS vidicons we have used in various versions of the Model-2 camera.

\section{TABLE I}

\section{MODEL-2 FPS CAMERA VIDICONS}

\begin{tabular}{|c|c|c|}
\hline Type & Target & Special Features \\
\hline Z7873 & $\mathrm{Sb}_{2} \mathrm{~S}_{9}$ & \\
\hline Z7873X & $\mathrm{Sb}_{2} \mathrm{~S}_{3}$ & $\mathrm{X}$-ray version of $\mathrm{Sb}_{2} \mathrm{~S}_{3}$ tube \\
\hline Zziz873S & $\mathrm{Si}$ & \\
\hline Z7801 & $\mathrm{Sb}_{2} \mathrm{~S}_{3}$ & POW, deflection plates printed on wall of tube \\
\hline Z7801S & $\mathrm{Si}$ & As previous \\
\hline 27803 & $\mathrm{Sb}_{2} \mathrm{~S}_{3}$ & \\
\hline Z780:3S & $\mathrm{Si}$ & \\
\hline Z7873FO & $\mathrm{Sb}_{2} \mathrm{~S}_{\mathrm{s}}$ & $\begin{array}{l}\text { IV, fiber-optics faceplate coupled to second- } \\
\text { generation microchannel-plate image intensifie }\end{array}$ \\
\hline $\mathrm{Z7873 \textrm {FO }}$ & $\mathrm{Sb}_{2} \mathrm{~S}_{\mathrm{a}}$ & $\begin{array}{l}\mathrm{I}^{2} \mathrm{~V} \text {, as previous plus cascaded proximity- } \\
\text { focused diode intensifier }\end{array}$ \\
\hline Z7803/4804 & Si & SIT, silicon intensified target \\
\hline $\mathrm{Z} 7803 / 4804$ & $\mathrm{Si}$ & $\begin{array}{l}\text { ISIT, SIT plus cascaded proximity-focused or } \\
\text { electrostatically focused diode intensifier }\end{array}$ \\
\hline
\end{tabular}




\section{CIRCUIT DESCRIPTIONS}

Figure 4 is a block diagram that is representative of both the Model-1 and -2 cameras, although the individual circuits are different for the two models. The camere head ans: camera-control unit (CCU) are separate chassis. The camera head contains the tube, tube-biasing network, focus coil, video preamplifier-amplifier, deflection amplifiers, filament-overvoltage protector, sweep-failure detector, and adjustments (focus, position, and astigmatism) for the electron optics. Most of the $\mathrm{CCU}$ volume is taken up by power supplies, but it also contains the sync and sweep generators, the catho le-blanking and sweep-protection circuits, and video processor-output amplifier circuits. The motor-driven potentiometers on the focus-, beam-, and target-voltage power supplies are used for adjusting these camera parameters remotely so the cameras can be used in environments inaccessible or hostile to experimentalists. The switrh-selectable rate-adjust and sync-level-shifter circuits were not included in the Model-2 FPS camera. Rate adjustment in that camera can be accomplished by simple rewiring.

\section{A. Video Preamplifier-Amplifiers}

We use one of two different video preamplifieramplifier (preamp) circuits, depending on the application. The two circuits have different frequencyresponse curves (Bode plots) and signal-to-noise ratios $(\mathrm{S} / \mathrm{N})$.

We use a moderate-bandwidth (14 $\mathrm{MHz}$ between -3 - $\mathrm{dB}$ points $)$, high- $\mathrm{S} / \mathrm{N}(\approx 300)$ preamp when horizontal resolution requirements are not severe. The high $\mathrm{S} / \mathrm{N}$ of this unit gives us excellent dynamic range in the final video. The preamp is based on an older commercial unit ${ }^{*}$ that is modified $^{7}$ for wider bandwidth and better $\mathrm{S} / \mathrm{N}$ (or dynamic range).

When wider bandwidth is required, we use the preamplifier-amplifier whose schematic diagram is shown in Fig. 5. The unit was designed to have a 30$\mathrm{MHz}$ bandwidth with optimum $\mathrm{S} / \mathrm{N}$.** The design is straigh.forward. The transimpedance-configured preamplifier stages consist of a low-noise-JFET

*The preamp referred to is that used in the Dage/MTI, Inc. VC20 Series cameras.

**The basic preamp desige was done by D. R. LiButti of EG\&G Inc. Energy Measurements group. source follower, a cascode gain stage, and an emitter follower, with overall feedback from the emitter follower to the JFET gate. The preamplifier is followed by a $20-\mathrm{MHz} \mathrm{RC}$ filter, a cascode gain stage $\left(\mathrm{A}_{\mathbf{v}}=\right.$ 15), and an output driver. In its present form, the midband transimpedance of the entire circuit is about $600 \mathrm{mV} / \mu \mathrm{A}$ with a $26.7-\mathrm{k} \Omega$ feedback resistor and a $50-\Omega$ load. The circuit will drive $\gtrsim 200 \mathrm{mV}$ p-p into a $50-\Omega$ load with less than $1 \%$ total harmonic distoration. The peak signal-to-rms noise ratio is therefore about $34 \mathrm{~dB}$, giving the camera a large potential dynamic range for its bandwidth.

Figure 6 shows theoretical and experimental transimpedance frequency-response curves for the $30-\mathrm{MHz}$ preamp. The dotted theoretical curve was obtained using the computer-aided design program NET-2. The experimental data were obtained from a spectrum-analyzer sweep, using a homemade passive current-source simulator driven by a tracking generator synchronized with the spectrum analyzer.

The experimental bandwidth of the preamp runs from about $25 \mathrm{kHz}$ to $30 \mathrm{MHz}$ at the $-3 \mathrm{~dB}$ points. The lower cutoff frequency is determined by the capacitive current divider formed by the input coupling capacitor and the effective parallel capacitance of the vidicon, its (short) connecting cable, and stray capacitance. We maximized the dynamic range by fixing the lower cutoff frequency at a relatively high value to virtually eliminate $1 / \mathrm{f}$ and other low-frequency noise. If this relatively high lower cutoff frequency causes excessive shaciing in the displayed picture, we eliminate the problem by using a distribution amplifier* that contains highquality fast dc restorers. The resolution in some cases can be degraded slightly when using this amplifier because its bandwidth is limited to about $20 \mathrm{MHz}$.

The relatively high peaking in the preamp (see Fig. 6) helps extend the overall camera frequency response. It tends to compensate both the rolloff from the input capacitance of the vidicon tube and the rolloff from the video processor that follows the preamp. The additional peaking results from stray inductance and capacitance imperfertly modeled by us when we used NET-2. We were not interested in producing a perfect theoretical model, but only in verifying the basic design and in quickly evaluating various design changes.

*The distribution amplifier was designed and packaged by $\mathrm{J}$. Shipley, G. Yates, and R. Dorsey of Los Alamos Scientific Laboratory. 


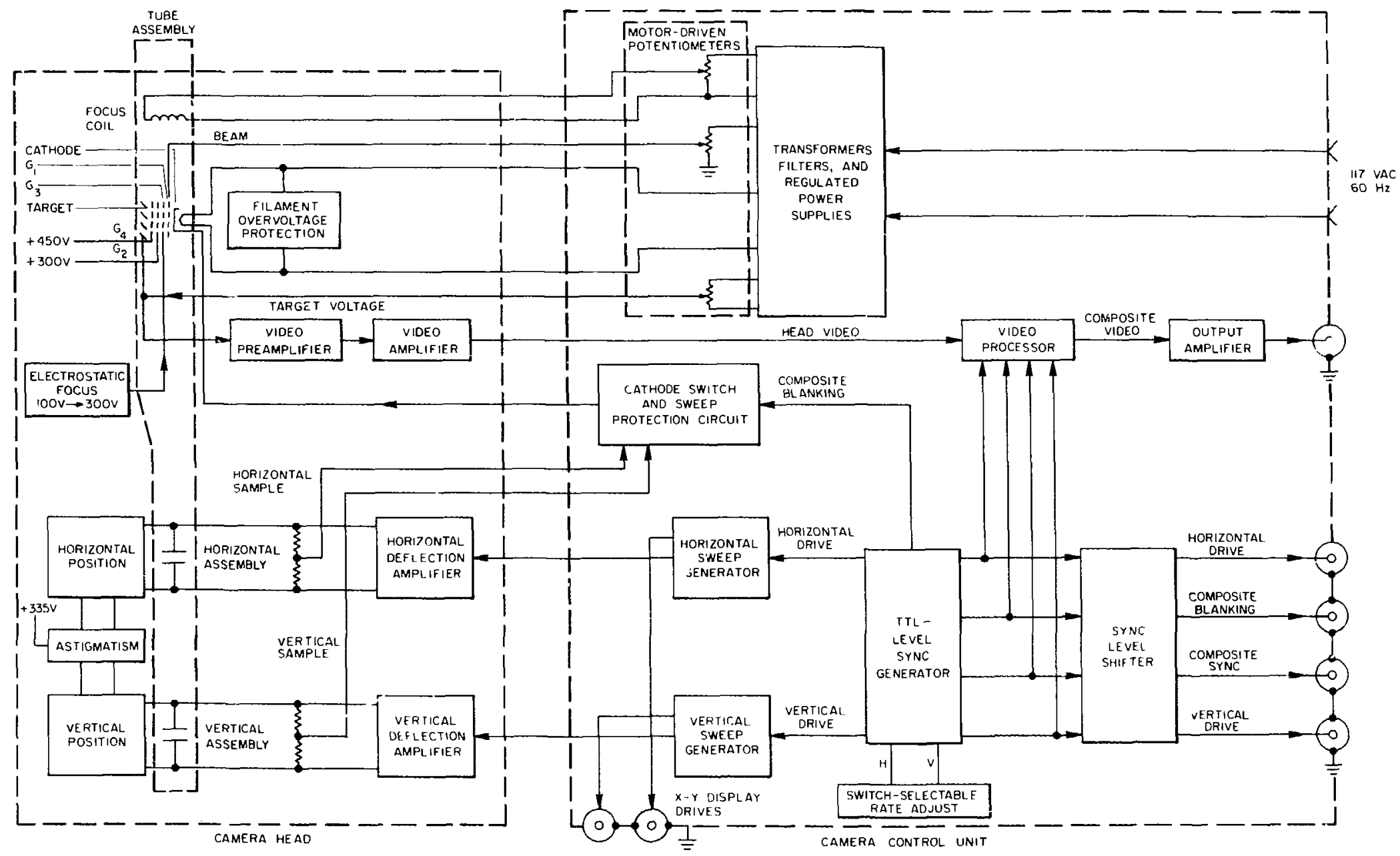

Fig. 4.

Simplified block diagram of Model-1 and -2 FPS cameras. 


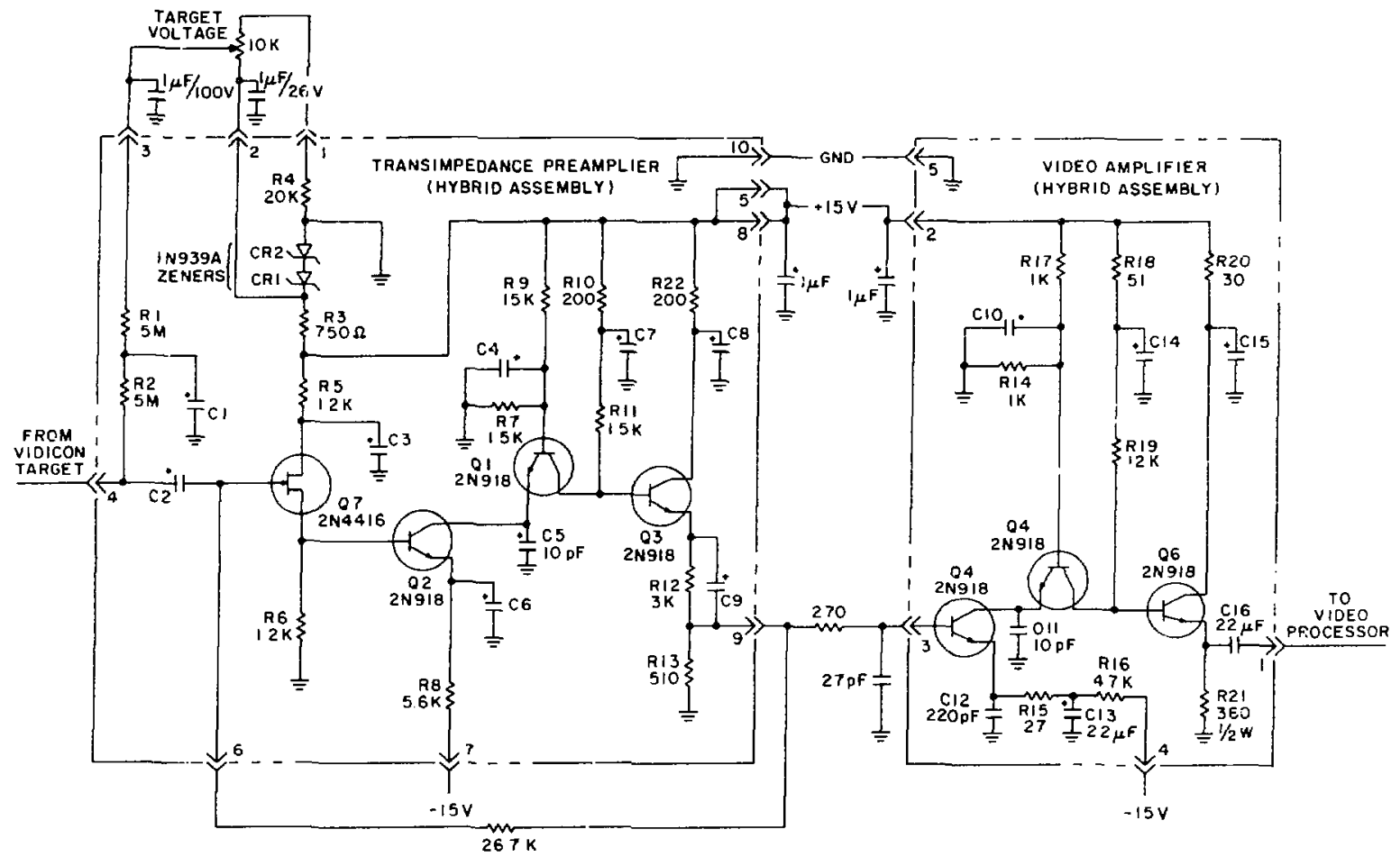

Fig. 5.

Schematic of the hybricized version of the 30-MHz video preamp.

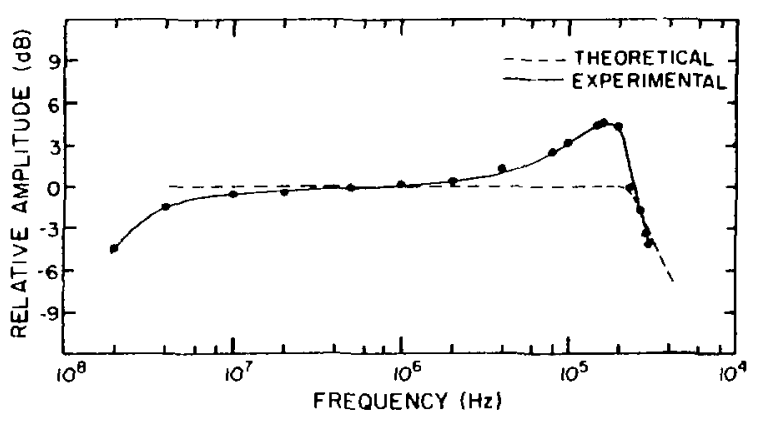

Fig. 6.

Theoretical and experimental frequency. response curves for the $30-\mathrm{MHz}$ preamp.

No phase-delay compensation, shading, or aperture correction circuits were included in either the preamp or video processor of the simple Model-1 or -2 camera prototypes. We plan to include these in a later prototype.
Figure 7 is a theoretically calculated nomogram that we can use to relate the essential parameters of the Model-2 camera for a given application. These parameters are (1) the desired field time in milliseconds; (2) the resolution (assuming that the horizontal and vertical resolutions are equal, that the vertical resolution is about 0.35 times the number of TV lines, and that the field time is the product of 1.1 , the line time, and the number of lines) in TV line pairs (TVLP) per picture height or in maximum line pairs (LP) per picture width; and (3) the required bandwidth in megahertz. The present limits of the Model-2 camera are indicated. Using the momogram we find that for our shortest presently available field time, $2.5 \mathrm{~ms}$, we should obtain about $460 \mathrm{TVLP} /$ picture height (nr LP/picture width) at $5 \%$ modulation by using the $30-\mathrm{MHz}$ preamp. Note that the nomogram was made using the manufacturer's data for $\mathrm{Sb}_{2} \mathrm{~S}_{3}$ tubes. For 460 TVLP/picture height, we need 460 TVLP over the $12.5 \mathrm{~mm}$ target height, or $37 \mathrm{LP} / \mathrm{mm}$ on the target, using the entire 

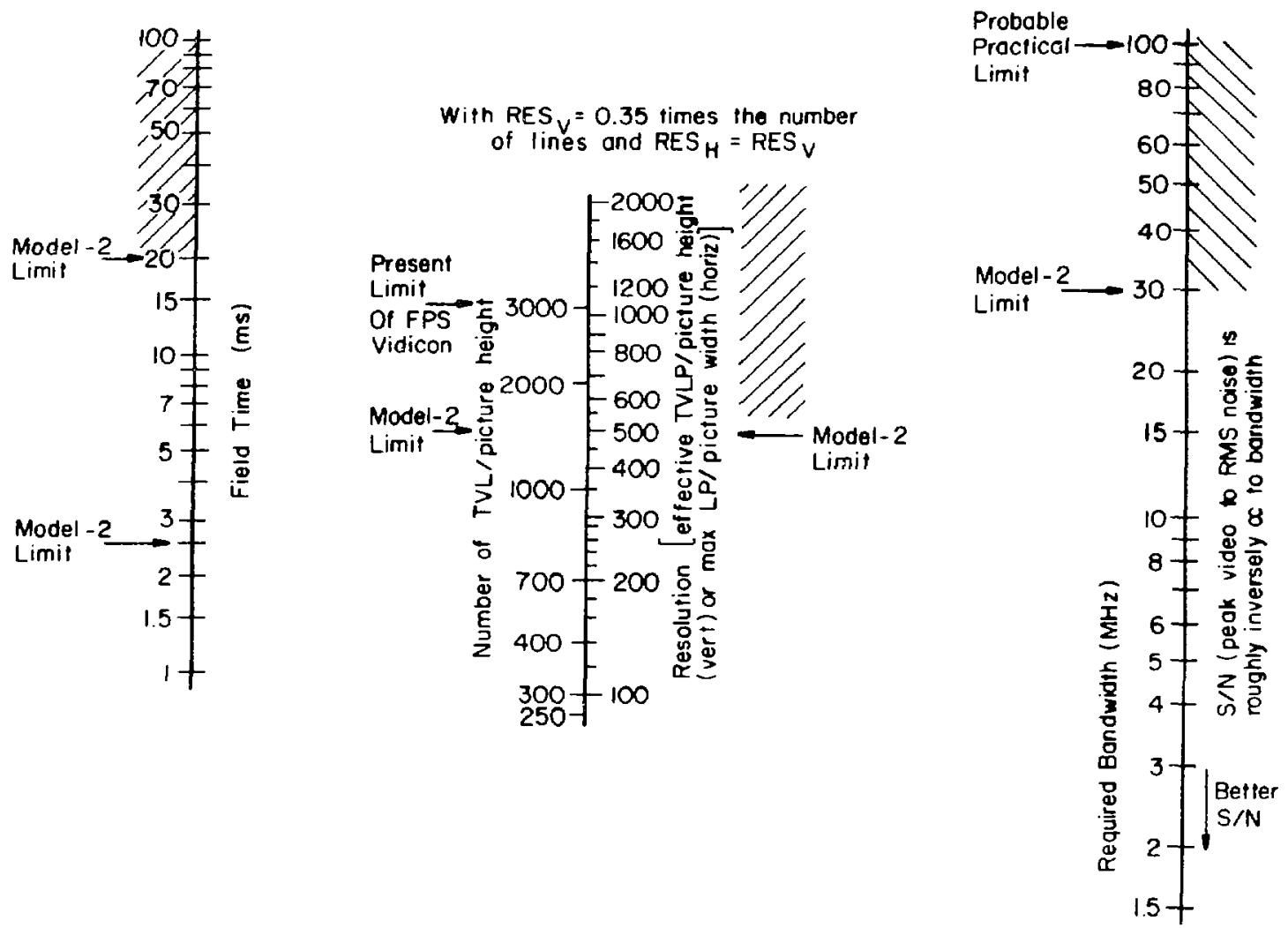

Fig. 7.

Nomogram relating field time, resolution, and preamp bandwidth for the Model-2 camera.

target height. This is approximately the resolution our cameras have achieved to date. Note also that it is not possible to achieve this resolution with silicontarget tules (assuming the manufacturer's data), so for a silicon-target tube, the parameters would have to be recalculated.

\section{B. Video Processors}

The Model-1 camera's video processor was basically a commercial unit extensively modified to permit its use in data-acquisition applications. ${ }^{7}$ Its features include a dynamic range compatible with that of the $14-\mathrm{MHz}$ preamp, output voltage swing of about $3.5 \mathrm{~V}$ p-p into $50 \Omega$ (not including sync and pedestal), nearly monotonic (that is, no overshoot) step-function response, ${ }^{*}$ and a temperature-stable pedestal network.

The first video processor used in the Model-2 camera was designed specifically for that camera.** The circuit diagram appears in Fig. 8. Its features include selectable signal polarity, independently adjustable pedestal and sync levels relative to the signal level, and the ability to drive $\gtrsim 6 \mathrm{~V} \mathrm{p}$-p into a long $(\approx 1 \cdot \mathrm{km}) 50 \cdot \Omega$ coaxial cable at $30-\mathrm{MHz}$ bandwidth. The output voltage swing can be improved to $210 \mathrm{~V}$ p-p by adddng the sync and pedestal with circuits that eliminate the isolation resistors. Alternatively, we could use the amplifier's

-This was done despite the loss of some bandwidth and of some phase-delay linearity because the need for a monotonic response dominated in our applications at that time.

"The basic design was done by $\mathrm{E}$. Wilson, formerly of EG\&G lnc. 


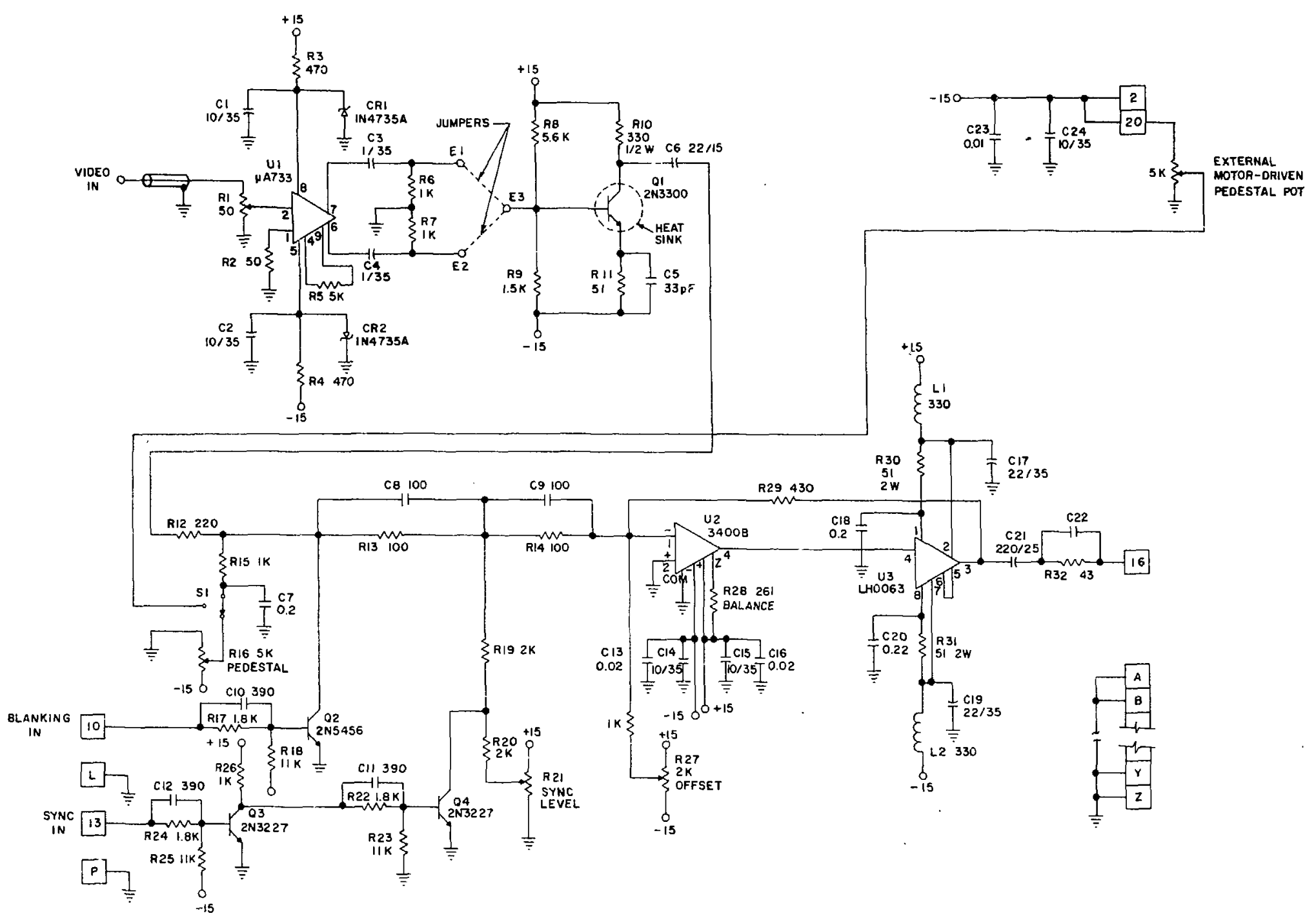

Fig. 8.

Early version of video processor for Model-2 camera. 
range for video only, adding the sync and pedestal after the output driver.

The circuit diagram for another video processor used in the Model-2 camera is shown Fig. 9. This video processor needs only one adjustment-for pedestal-since the sync-tip level is fixed relative to the blanking level. It uses only an external motordriven pedestal potentiometer, since the cameras in which it is used are always operated remotely. For a typical preamp input signal amplitude of $60 \mathrm{mV} \mathrm{p}$-p, the output video is $4 \mathrm{~V} p-p$, exclusive of sync and pedestal.

\section{Sync Generators}

The basic sync generators for the Model-1 and -2 cameras are similar. The designs are both all-digital. The one major difference lies in how they drive the sweep generators. The Model-1 camera used analog sweeps, but the Model-2 camera can use either digital or analog sweeps. The digital sweep generators use digital-to-analog converters (DACs). The sweep direction can be controlled in the Model2 camera because its sync generator uses type 74193 synchronous up/down counters; type 7493 synchronous up-only counters were used in the Model-1 camera. The 74193 counters minimize glitches in the DACs by minimizing time skew (phase differences) between supposedly concurrent bit-level changes at the $\mathrm{DAC}$ input register. DACs with built-in deglitching or a combination of DACs and deglitchers will be used in future designs.

The Model-2 sync generators contain four main parts, as shown in Fig. 10. The $20-\mathrm{MHz}$ crystaloscillator system clock A1-1 feeds two sets of rounters. One set, a cascade of four decade counters (A1-2 through A1-5), forms a precision delay circuit. When triggered externally, perhaps by an event time-related to the expected data, this circuit resets the remaining counters, synchronizing the $\mathrm{n}$ with a similar sync system at the data-recording itation. Alternatively, for master-slave operation the reset lines can be driven by an external sync generator's vertical pulse train.

The other set of counters generates the horizontal and vertical sync waveforms.
The horizontal counters A2-1 through A2-3 are fed by a chosen clock frequency. The horizontal period is further trimmed by summing chosen outputs of the counters' registers with $\mathrm{AND}$ gate $\mathrm{G}_{1}$. The Model-1 camera used 8PST DIP switches for these choices. The manual switches were deliberately eliminated in the Model-2 camera to allow for future automated control (described below). Jumpers were used instead. The Model-2 sync generators are fabricated on $\mathrm{CASH}^{*}$ Wire-Wrap ${ }^{* *}$ boards where selection by jumpers is easily done.

The vertical counters A3-1 through A3-3 are clocked by the horizontal counters' output pulse train and count 256 pulses. An additional eight pulses are counted to generate the vertical sync interval. Both horizontal and vertical counters are then reset and a new cycle begins.

The sync- and blanking-pulse widths are generated digitally by summing the appropriate counter outputs.

\section{Sweep Generators}

We have designed and used several different types of sweep generators. Some are analog and some are digital. All feature adjustable line and field durations. In the basic versions, the rates are manually adjustable. In the remotely controllable versions, the rates, number of scan lines per field, and integration duty cycle ${ }^{+}$may be adjusted at will by remote controls.

Two other types of sweep zenerators are being developed but are not discussed in detail here. One of these is a "homing" raster generator that looks for a point on the target where the data starts and generates a raster only across the data. Another type uses variable-slope ramps to decrease the beam sweep velocity in regions of expected high spatial density of information and to increase the velocity elsewhere. Selectively decreasing the velocity produces lower electronic frequencies that are easier for limited-bandwidth amplifiers to handle. It may

* CASH is a registered trademark of Standard Logic, Inc.

**Wire-Wrap is a registered trademark of Gardrier-Denver Co.

tWe define the integration duty cycle as the ratio of the number of lields read out to the total number of fields, including those during which charge is integrated on the target. 


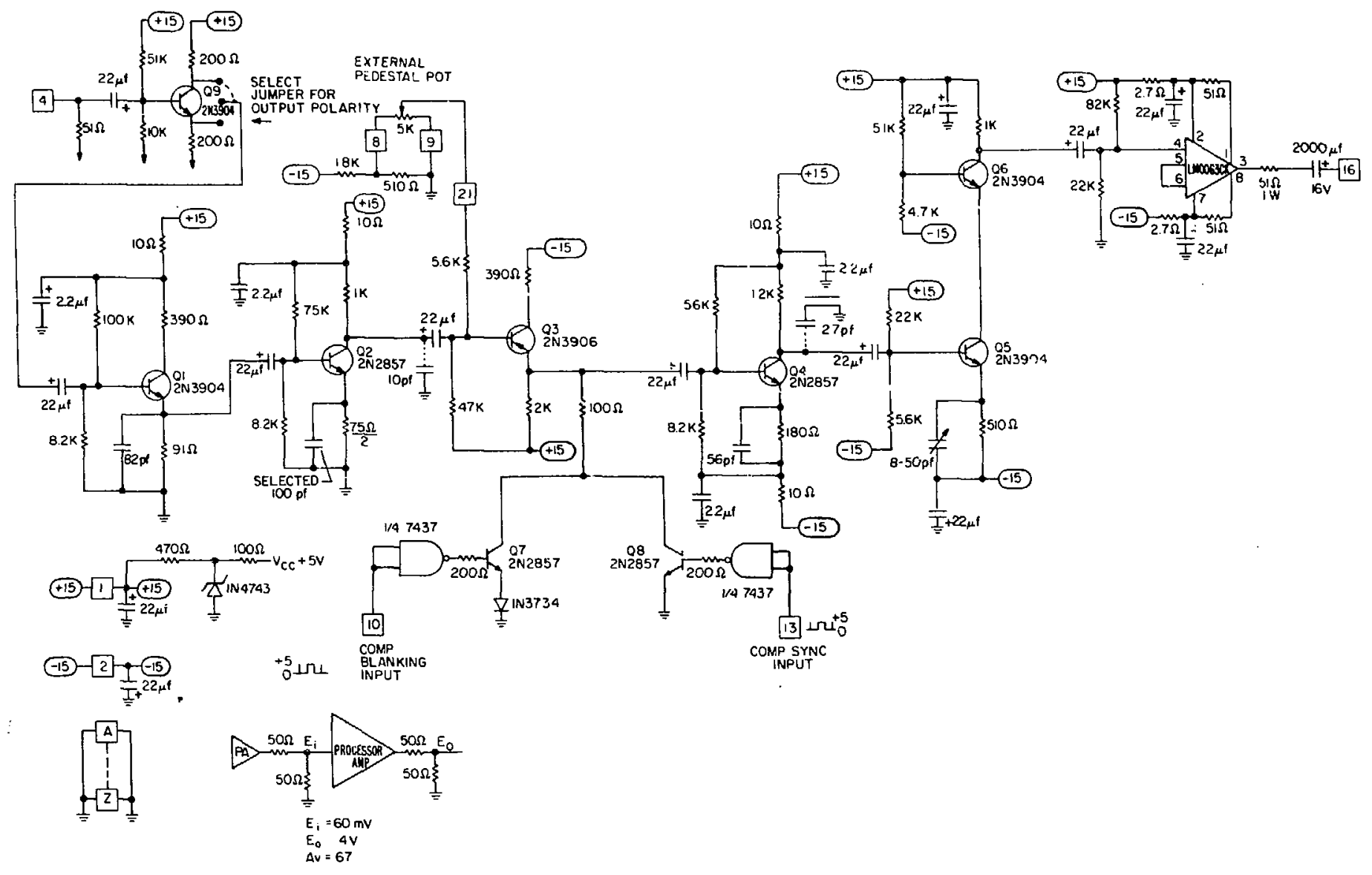

Fig. 9.

Later version of video processor for Model-2 camera. 


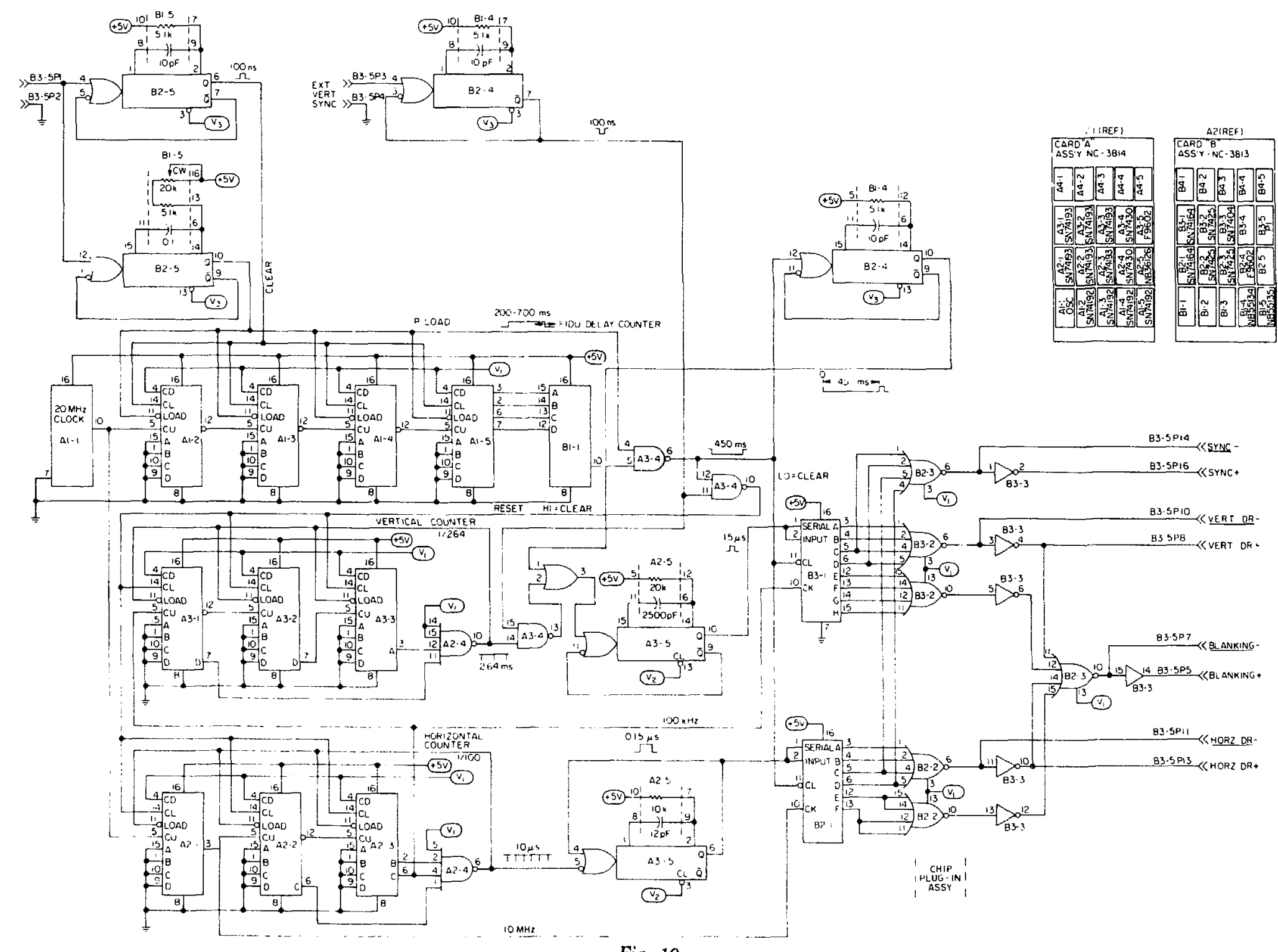


or may not improve the $\mathrm{S}_{i} \mathrm{~N}$ also: a narrowerbandwidth amplifier can give lower noise, but slowing down the beam decreases the signal current because of the $\Delta Q / \Delta t$ effect described above.

1. Basic Analog Sweep Generators. Linear ramps for the analog sweers for both camera models were generated by intes ating a constant-current source for the respective line or field period. Figure 11 shows the basic sweep circuit. The vertical and horizontal integrator designs are identical except for their RC time constants, which were selectable in discrete combinations of $\mathrm{R}$ and $\mathrm{C}$ by either DIP switches or jumpers. This method of selecting time constants discretely provided goad repeatability that was useful in evaluating camera performance at various oweep rates. The horizontal and vertical drive signals from the sync generator discharge the integration capacitors through MOSFET switches, thereby ending the integration period and initiating the next cycle.

\section{Remotely Controllable Analog Sweep} Generators. In this version the Model-2 camera's sync generator and sweep generator are designea to permit the user to select or alter, by remote control in response to time-changing data, the scan-line duration, the field duration, and the integration duty cycle. The selectability feature was added to fulfill the needs of a particular application discussed below. The user can select any one of four line durations: $8,16,32$, or $64 \mu \mathrm{s}$. The number of scan lines per field was set at 256 . Thus the field durations are $2.048,4.096,8.192$, and $16.384 \mathrm{~ms}$. The variable integration duty cycle feature permits reading either consecutive fields ( $100 \%$ duty cycle) or every second, third, or fifth field.

The peak sweep amplitudes are held constant to eliminate position and size jitter in the raster when the line durations are changed. This required using very large capacitors to ac-couple the ramps to the deflection amplifiers to minimize changes in the raster position.

A simplified schematic diagram is shown in Fig. 12. Drivers, inverters, and similar components are not shown.

The desired line rate is selected by gating the proper pair of capacitors (one for the vertical rate and one for the horizontal rate) into the feedback path of the integrators and changing the horizontal period by changing the input clock frequency. Each integrating capacitor is in series with a Teledyne 640-1 solid-state relay ( $\mathrm{K} 1 \mathrm{H}$ through $\mathrm{K} 4 \mathrm{H}$ and $\mathrm{K} 1 \mathrm{~V}$ through $\mathrm{K} 4 \mathrm{~V}$ ). The integrating capacitor is selected by closing its associated series relay. The relays are operated in horizontal-vertical pairs to maintain the fixed line count per field and the constant sweep amplitude mentioned above. The horizontal clock frequency is selected by th:e 4-to-1 multiplexer, IC2. At any frequency, the horizontal counters (ICs 6-8) count the same number of pulses to generate the

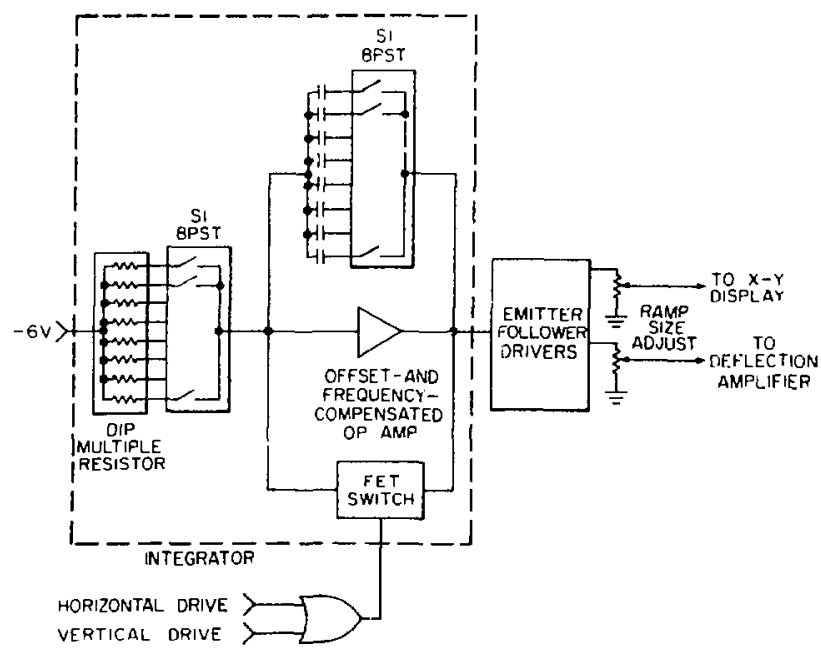

Fig. 11.

Variable-rate analog sweep generator. S1 and S2 are DIP-compatible 8PST passive switches. 


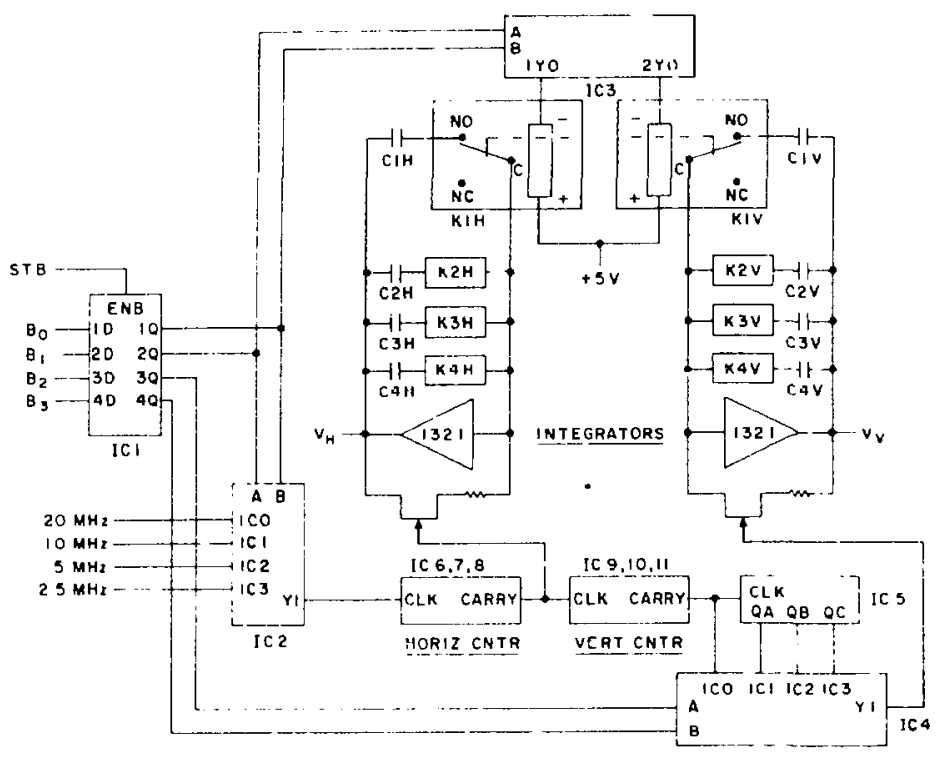

Fig. 12.

Simplified schem atic of the remotely controllable analog sweep generator.

desired period. At the end of the period, the horizontal integrator's capacitor is discharged, generating the retrace portion of the sweep.

The vertical counters (ICs 9-11) count 256 of the horizontal periods. Then a vertical drive pulse is generated that is used to discharge the vertical integrator, thus ending the sweep ramp and initiating a new cycle.

After the field-readout period is selected, the integration duty cycle can be set. Another 4-to-1 multiplexer, IC4, selects either the output of the vertical counter or one of the outputs of the separate counter, IC5, that counts field pulses. In this way the vertical integrator circuit is held disabled for either the normal vertical interval, or for 1,2 , or 4 field periods.

The two 4-to-1 multiplexers and the type 741561 to-4 demultiplexer (IC3) are controlled or biased by the dual-purpose latch, IC1. This latch is wired to perform as two pairs of strobed D-type flip-flops. One pair, 1D and 2D, serves as a field-period latch that operates the select lines of the 4-to-1 multiplexer and the 1-to-4 demultiplexer that control the field-period selection circuits. The other pair, 3D and $4 \mathrm{D}$, serves as a duty-cycle latch that operates the 4-to-1 multiplexer that controls the duty cycle.
Although the data inputs of the latches can be controlied manually with static biases, they are normally computer-controlled. The computer sets the bit pattern on the data inputs in some predetermined way in response to the (pernaps randomly) changing intensity of the scene being viewed by the camera. Because either th? field-readout period or the integration duty cycle can be changed quickly by strobi ig new data into the latch, the camera's rosolution and sensitivity can be changed spontaneous!y by the user to best meet the requirements of changing scenes.

\section{Remotely Coytrollable Digital Sweep} Generator. This powerful, versatile subsystem provides almost romplete control over the Model-2 camera's raster It is intended as a laboratory tool for examining strategic locations on the FPS vidicon target with maximum flexibility. It is on a separate chassis that plugs into a CAMAC crate to provide easy control and formatting of the rasters from either a programmed manual crate controller or from a PDP-11/34 (with UNIBUS* adapter) driving

${ }^{*}$ PDP and UNIBUS are registered trademarks of Digital Equipment Corp 
the CAMAC dataway. The sweep formats can also be programmed manually from front-panel controls on the CAMAC chassis. The controllable raster parameters include line and field periods, number of lines, and start-stop coordinates and scan directions for both scan axes.*

The digital sweep system gen $€ \quad 39$ ramps by addressing fast-settling DACs. Th JAC settling time per bit must be short enough to permit ultrafast clocking so that high-quality ramps can be cormed at even the highest sweep rates. A Datel model DACHIIOB, a 10-bit fast-settling (worst case: $25 \mathrm{~ns}$ to $0.1 \%$ full scale) current-output unit can be used for horizontal line durations as short as $6.4 \mu \mathrm{s}$. An even faster-settling (20 ns to $0.1 \%$ full scale) currentoutput DAC, Computer Labs model MDS-1020, is used in the camera version that provides $5.12-\mu \mathrm{s}$ horizontal line durations. These line times are achieved using the lowest 8 bits to provide scan lines with horizontal resolution of 256 steps. The 10-bit capability permits fut:ure expansion to 1024-step horizontal and vertical resolution.

The digital sweep system, a simplified circuit of which is shown in Fig. 13, contains a master urithmetic logic unit (ALU) composed of three connected 74181 4-bit ALUs, ICs 13-15. The master

- Zoom can be added with some circuit modifications.
ALU, which also is controlled by either a computer or from the front panel, provides a fast, powerful tool for operations requiring higher speed than is attainable by sequential instruction sets from the PDP-11/CAMAC system. The master ALU con perform 16 arithmetic operations on two 10-bit operands. The operations include addition, subtraction, shift operand $A$ one positior. (decrement), and magnitude comparison. Alternatively, it can provide 16 logic functions instead of the arithmetic functions, including among others EXCLUSIVE OR, AND, NAND, OR, NOR, and digital compare.

The DAC's input registers are driven by the buffered outputs of programmed synchronous up-down counters that use 4-bit binary 74193 counters, ICs 7 9 . The count direction is controlled by enabling the up-clock input while disabling the down clock, or vice versa. The counter data-input lines can be dynamically or manually preloaded to enter start. coordinate values for either DAC. Stop-coordinate values are entered into a second identical set of counters, ICs 10-12. The ALU digitally compares the DAC's current bit value with the stop value. When the two are equal, the desired raster dimensions have been generated and the next identical cycle is initiated automatically unless new conditions are loaded into the counters.

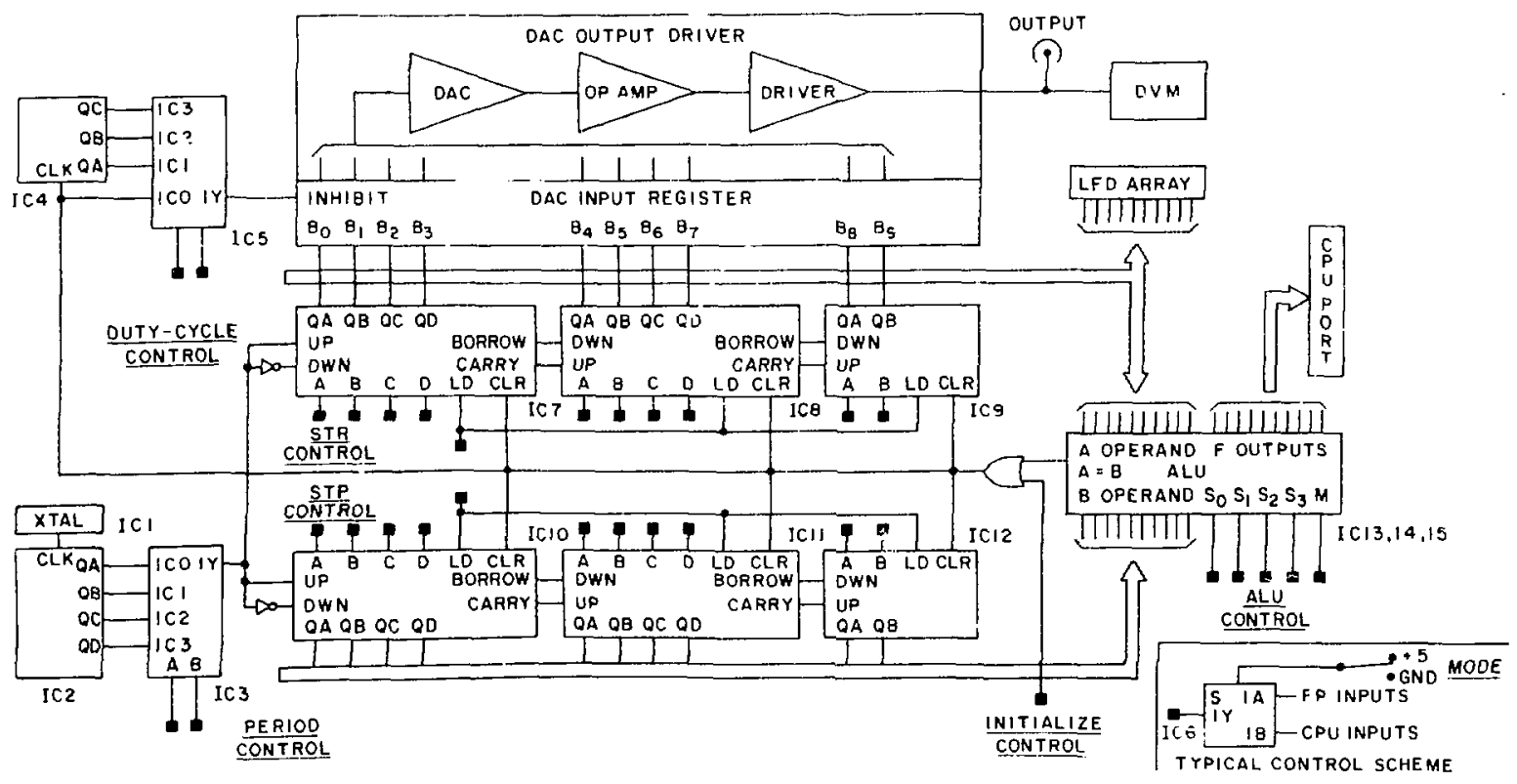

Fig. 13.

Simplified schematic of the remotely controllable digital sweep generator. 
The DAC outputs are fed to the low-impedance summing junction of a Teledyne Philbrick 1322 high-slew-rate operational amplifier (op amp), which was used to keep the overall response time small and to boost the ramp amplitude to the proper level.

The system includes both analog and digital readouts of the current value of the DAC word. These readouts are used, for example, to monitor end-point and midpoint drifts of the DAC/op-amp dynamic range and to indicate slowly changing bit patterns. The digital readout is a row of LEDs that sense the DAC inpL, register. The analog readout uses an Analog Devices model 2010 DVM that samples the analog output of the DAC-driven op amp and provides a four-digit decimal presentation of the sweep amplitude.

The readout speed is controlled either by selecting different clock frequencies from the crystal oscillator or from the subsequent counters. Gross control can be achieved during setup by changing the crystal to one of a different base frequency.
E. Deflection Amplifers. The Model-1 camera deflection amplifiers used only discrete components. A diagram of the deflection circuits is shown in Fig. 14. The horizontal and vertical amplifiers are identical. The push-pull output stages amplify the input-stage sweep voltage by a nominal factor of 8 $\left(2<A_{v}<10\right)$. The resulting sawtooth ramp amplitudes are 20 to $100 \mathrm{~V} \mathrm{p}$-p. The low-value load resistors provide relatively low output impedance to minimize the loading effect of the vidicon plate capacitance.

The deflection amplifiers for the Model-2 camera have the versatility to use a variety of FPS vidicons, some of which required larger deflection voltages than were available from the Model-1 amplifiers. The Model-2 amplifiers used a combination of hybrid integrated circuits and discrete components. Double lines in the schematic diagram shown in Fig. 15 separate the hybridized and discrete components. The horizontal and vertical amplifiers are identical. The two stages have sufficient gain and dynamic range to produce $180-\mathrm{V}$ ramps. The first stage of the

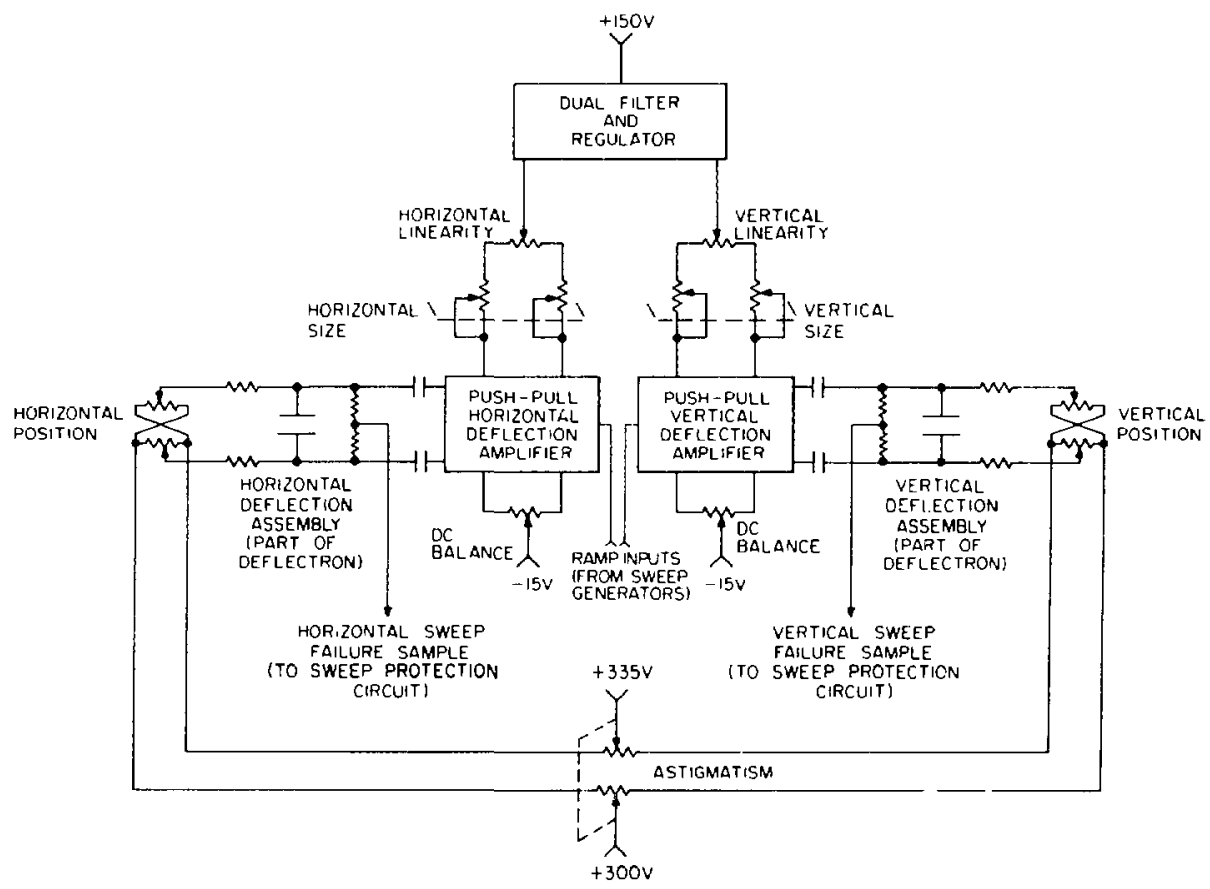

Fig. 14.

Deflection circuits for the Model-1 camera. 


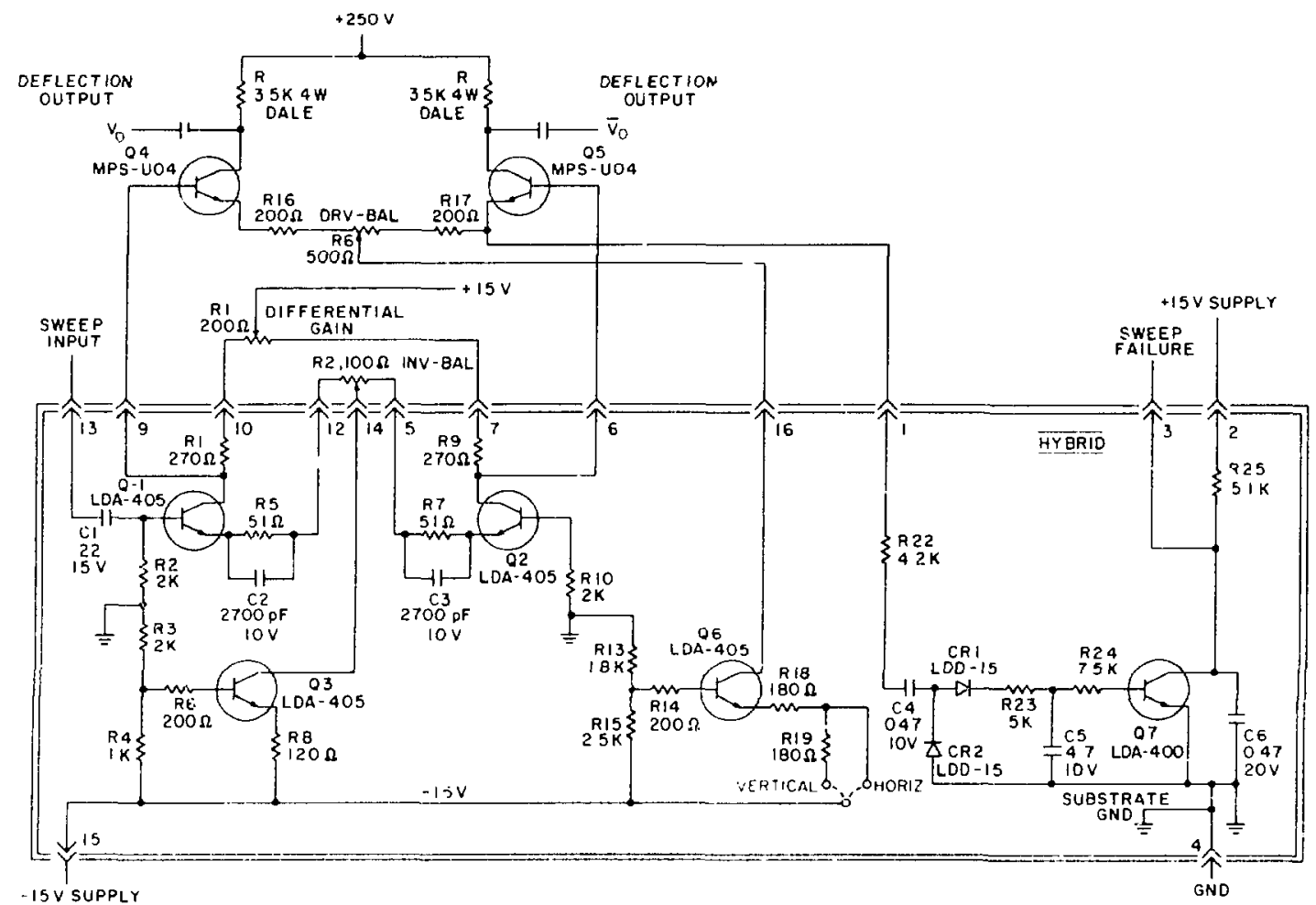

Fig. 15.

Defleciion-amplifier and sweep-failure circuits for the Model-2 camera.

differential pair $Q 1$ and $Q 2$ gives some voltage gain $\left(A_{v} \approx 2\right)$, but its primary function is to provide phase splitting. Q3 forms a bias-current source. A small amount of differential gain is provided by poten. tiometer R1. Both polarities of the input sweep waveform are available at the collector outputs, giving equal but opposite drive to the output transistor: Q4 and Q5.

The second (output) stage has a voltage gain $A_{v}=$ 15. The output signals are taken from the collectors of Q4 and Q5, a matched pair of Motorola MPSU04s. The bias current is supplied by the current source transistor Q6. Sharing of the bias current is supplied by the balance potentiometer R6. The ramp voltages are ac-coupled to the FPS vidicon deflectron-type deflection plates. The plates are referenced to $\approx 300 \mathrm{~V} \mathrm{dc}$ through the horizontal and vertical position potentiometers. This referencing keeps the deflection assembly at the proper voltage to conserve the internal electrostatic field required for proper operation of the electron-veam optics.
The sweep-failure detection and protection circuits are included in the hybrid integrated-circuit assembly. These circuits are in one leg of the emitter circuit of the output differential amplifiers. When no ramp is generated at the output, Q7 is biased off, giving a static bias that is mixed in a later circuit to provide composite blanking for the FPS vidicon cathode. The static bias gates off the tube's beam, thus preventing any 'burns' on the target caused by a stationary beam.

\section{F. Cathode-Blanking, Tube-Biasing, and Focus- Regulator Circuits}

The composite blanking waveform gates off the read beam during the line and field retrace intervals in normal operation and upon sweep failure. The waveform from the sync generator is fed to one input of an OR gate. The sweep-failure detection circuit is connected to the other input of the OR gate. The 
TTL-level input signal is converted by a highvoltage switch to a $30 . \mathrm{V}$ pulse that drives the vidicon cathode positive, thereby blanking the beam.

With one exception, the tube-biasing circuits are simply decoupling networks used to minimize exter"lal noise pickup and to stabilize the dc levels needed to operate the tube. The voltage on grid $\mathrm{G}_{2}$ (see Fig. 4) sets the maximum available bcam current. Because this voltage maintains the stability of $G_{2}$ under varying signal-current conditions, the $G_{2}$ bias network uses a current-source transistor that supplies a regulated voltage source for the grid.

The focus-regulator circuit shown in Fig. 16 supplies sufficient current to operate any of several different focus-coil assemblies (all with different impedances) in either the first or second focus node ${ }^{2}$ of the FPS tul \&. The current can be adjusted over the range $100-300 \mathrm{~mA}$ either manually or remotely by a motor-driven potentiometer. The circuit uses a

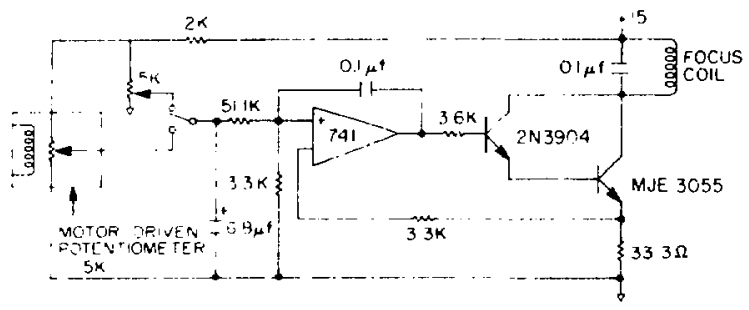

Fig. 16.

Focus-regulator circuit schematic. type 741 op amp in a feedback arrangement and a Darlington-coupled pair for en output driver. The focus coil is the output driver's collector load and the feedback resistor is in its emitter circuit.

\section{CONSTRUCTION DETAILS}

\section{A. Model-1 FPS Camera}

The Model-1 camera head and CCU (Fig. 17) were modifications of our EDR camera. The original head preamp was retained, but virtually all the other circuits were replaced, modified, or eliminated. The original CCU chassis width was reduced by onethird to make it more compact.

\section{B. Model-2 FPS Camera}

The Model-2 camera head and CCU were totally new designs. Both were designed to provide good $\mathrm{rf}$ shielding. The body of the camera head (Fig. 18) is milled from solid aluminum. In use, a large finned heat sink is bolted to and covers one side of the camera head. The exploded view in Fig. 19 shows the front lens-mount plate, which is removable for easy access to the tube and preamp. The plate has a double screw-in mechanism. The center hole in the brass disk is threaded to accept standard C-mount lenses. The disk screws into the front plate to provide coarse mechanical focusing. Figure 20 shows a thick-film hybrid integrated-circuit version of the $30-\mathrm{MHz}$ preamp that mounts in the front of the

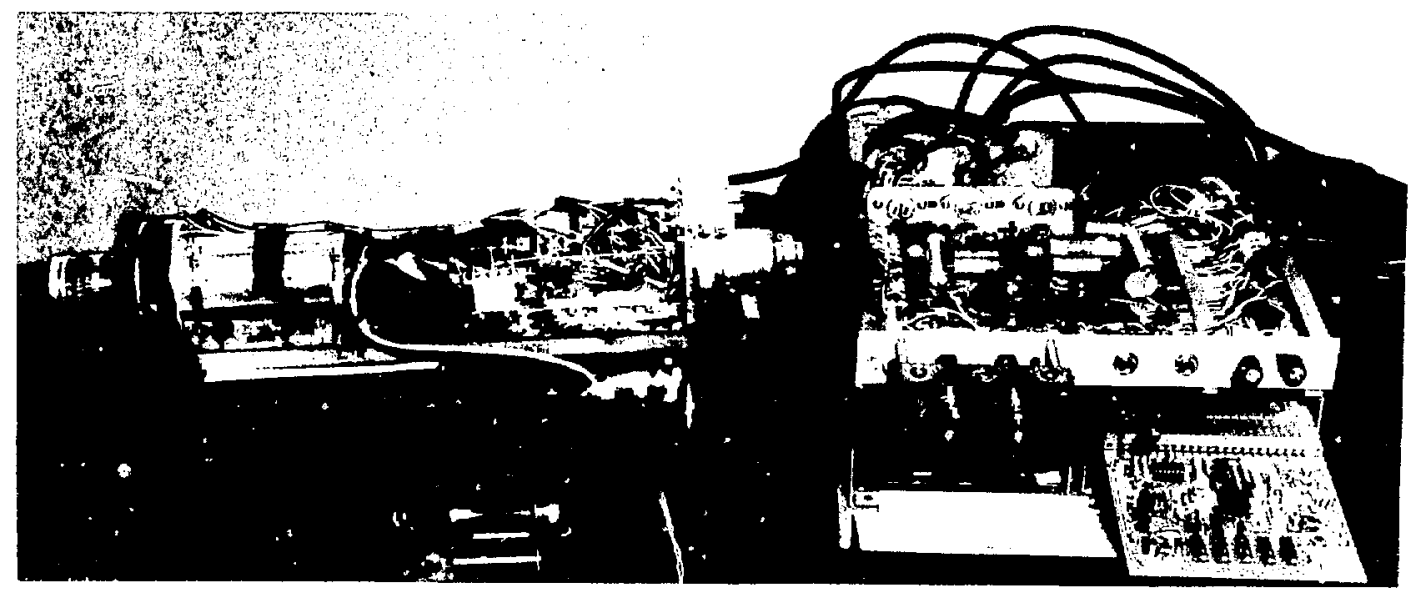

Fig. 17.

Photograph of the Model-1 camera head (at left, cover removed) and CCU (at right, top cover removed, access door open, and sync generator on extender card). 
camera, behind the lens plate and surrounding the vidicon face. One version of the rear of the camera head is shown in Fig. 21. The deflection amplifiers-exclusive of output transistors, load resistors, and balance and gain potentiometers-are fabricated in thick-film hybrid integrated-circuit form and mounted on a printed-circuit board. For better heat dissipation, the four MPS-U04 output transistors are mounted below the integrated circuits on the wall of the head. In an har version, Fig. 22, the transistors are mounted inside and the power resistors are mounted on the rear plate.

A top view of the CCU, with its cover removed, is shown in Fig. 23. Two Wire-Wrap digital cards at upper left comprise the sync generator. The analog sweep-and blanking-generator card is just below them. To the right, covering two of the seven power supplies, is a focus-regulator card. Figure 24 shows a bottcm view with \& plate removed to expose the bottom wiring on the sync-generator cards. The videoprocessor card is located here also. It and the two analog cards in the top portion of the chassis are hinged for access to their undersides for troubleshooting.

Front and rear views of the remotely controllable digit al sweep generator are shown in Figs. 25 and 26. In Fig. 25, the unit is shown partly installed in its st andard CAMAC crate.

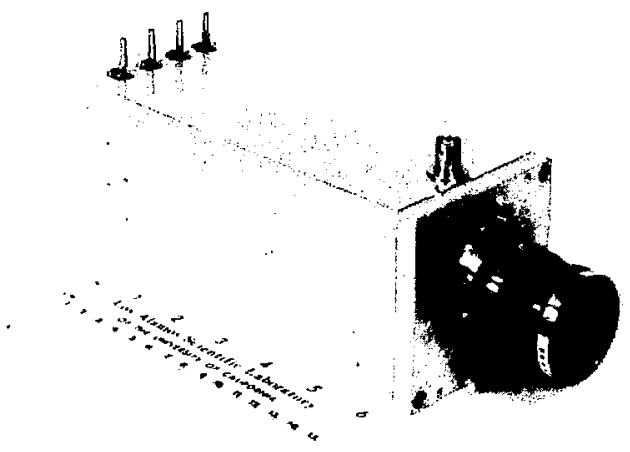

Fig. 18.

Photograph of Model-2 camera head without bolt-on heat sink.

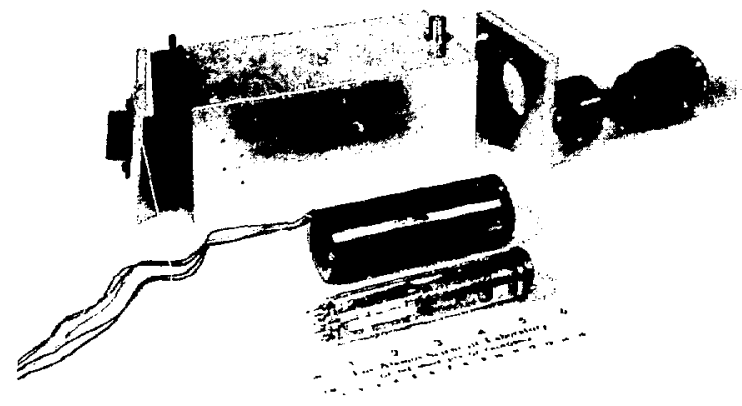

Fig. 19.

Exploded view of Model-2 camera head, showing FPS vidicon, focus coil, and lens assembly.

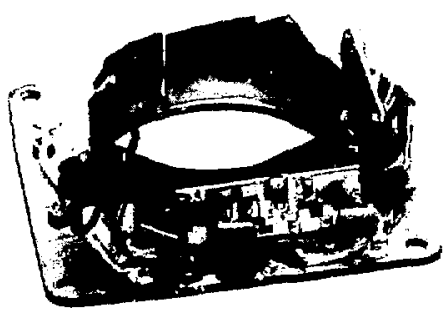

Fig. 20.

Video preamp board with two-section hybrid integrated-circuit preamp.

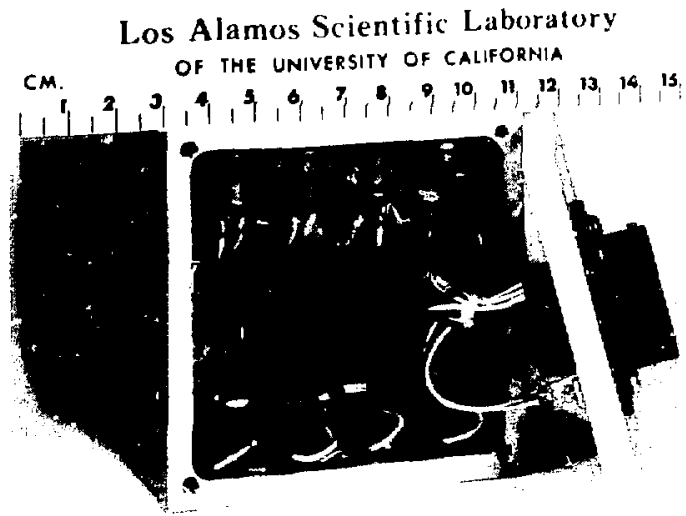

Fig. 21 .

Rear view of the Model-2 camera head, showing one version of the deflection-amplifier circuit. 


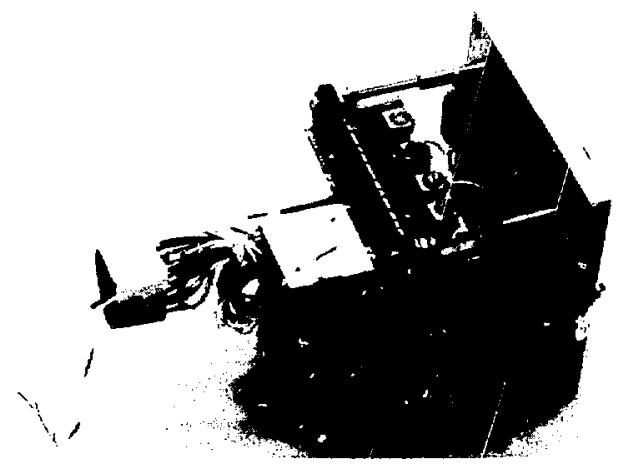

Fig. 22.

Another version of the Model-2 deflectionamplifier circuit mounted on the rear plate of the crmera head.

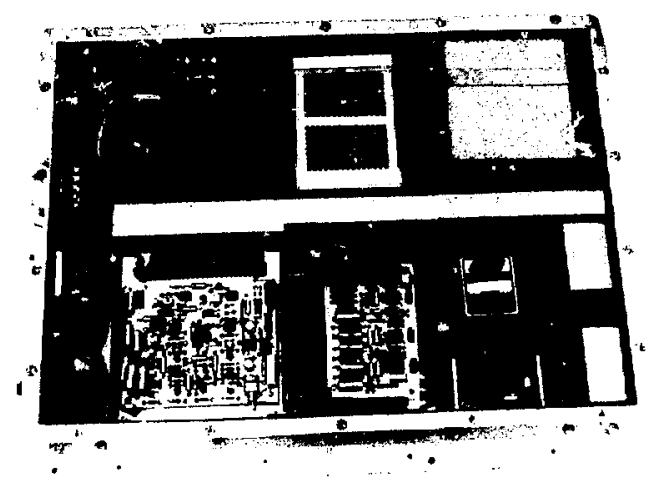

Fig. 23.

Top view of Model-2 CCU.

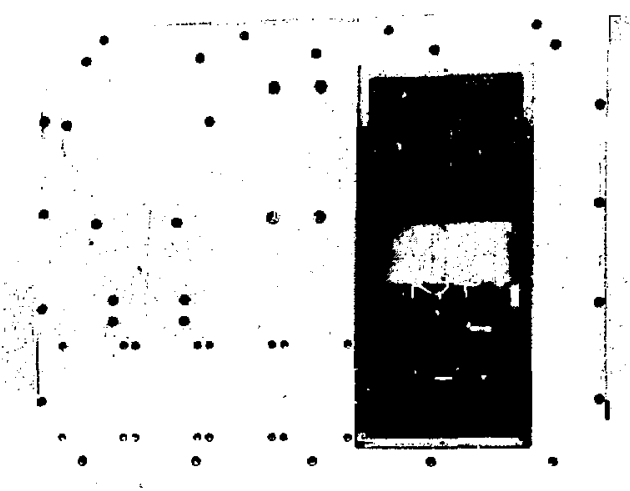

Fig. 24.

Bottom view of Model-2 CCU.

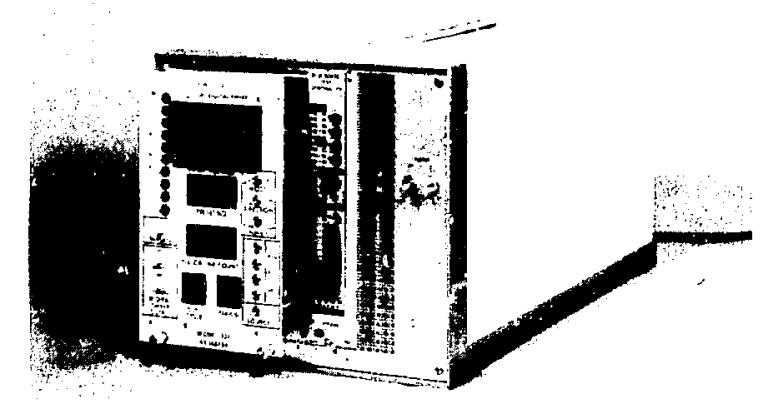

Fig. 25.

Front view of the remotely controllable digital sweep subsystem.

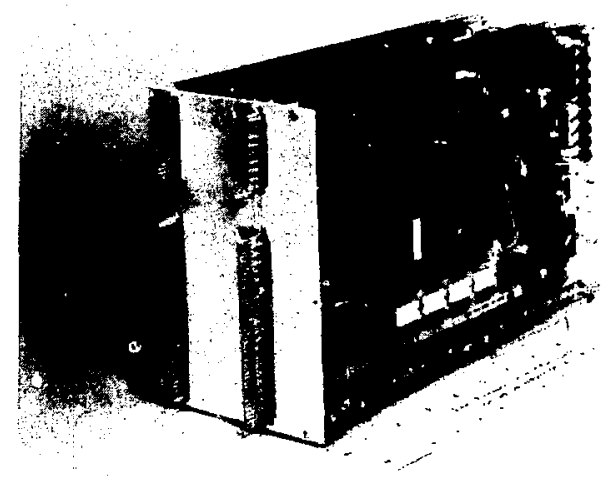

Fig. 26.

Rear and side view of the remotely controllable digital sweep subsystem. 


\section{CALIBRATIONS}

The low-frequency transfer characteristic is the most important calibration for any data-acquisition camera. Nearly as important is either the contrasttransfer function (CTF) curve or the modulationtransfer function (MTF) curve. The complete optical-transfer function includes the phase-vsfrequency curve as well as the MTF curve. Although the phase-vs-frequency and also the phase delay-vsfrequency curves would be very useful, we do not at present have the equipment for obtaining them. We obtain the transfer characteristic for every camera and often also obtain the CTF curve (from which the MTF curve can be obtained if desired). Most of the comments in the following two sections apply to any data-acquisition camera.

\section{A. Transfer Characteristics}

One can obtain low-frequency $\mathrm{cw}$ transfer characteristics using a television Optoliner.* Including a standard bar chart in the Optoliner gives the data for the CTF curve as well. We often use instead a pulsed light source, usually a GenRad model 1539-A Stroboslave that has been referenced to a standard light source. Reciprocity holds under most conditions; that is, the camera produces the same video output voltage for a pulsed source of any width down to at least $200 \mathrm{~ns}$ as it does for a de source, provided the input energy is the same. ${ }^{8}$ We display the video signai on an oscilloscope, select one or more horizontal lines, and read the output voltage from the display. The light amplitude can be varied by using a combination of camera lens $f$-stops** and neutral-density (ND) filters. The ND filters may be caiibrated accurately with a spectrophotometer. We place bond paper in front of the Stroboslave to diffuse the light and place in front of that a simple bar chart made by mounting a series of different widths of printed-circuit layout tape un clear Plexiglas. The Stroboslave's spectral output approaches that of a white source. For some types of experiments we find it useful to find the camera response per unit wavelength for blue light. For this purpose, we add a Corning CS5-61 narrowband blue filter to the lightsource assembly.

${ }^{*}$ A registered trademark of the Photo Research Division of Kollmorgen Corp.

**We have found many commercial lenses, however, .:ich the f/siops are not accurately calibrated.
The video output voltage for a given light input is a function of many variables, as is the dynamic range. We often find it helpful to obtain a family of transfer characteristics using one or more of these variables as a parameter. For example, the transfer characteristic shifts upward with an increase in beam current up to the lirit imposed by the maximum available beam current. An increase in scan rate shifts the transfer characteristic upwards until the scan rate is so high that not all the charge can be removed by the passing beam; the curve then shifts downward again. The transfer characteristic shifts upward as the integration time is increased; the limit is achieved when the integrated dark current equals or exceeds the signal (that is, ine $\mathbf{S} / \mathrm{N}$ drops to unity or below). This effect is illustrated in Fig. 27 , where the video output voltage is plotted vs integration time for a fixed light-input level. The $S / N$ is optimum up to about $16 \mathrm{~ms}$, where it starts to drop. The transfer characteristic also shifte upward as the target bias is increased up to a saturation point. It shifts upward as the snak time is increased to the point where soaking is finished. Of course, any transfer characteristic taken before soaking is

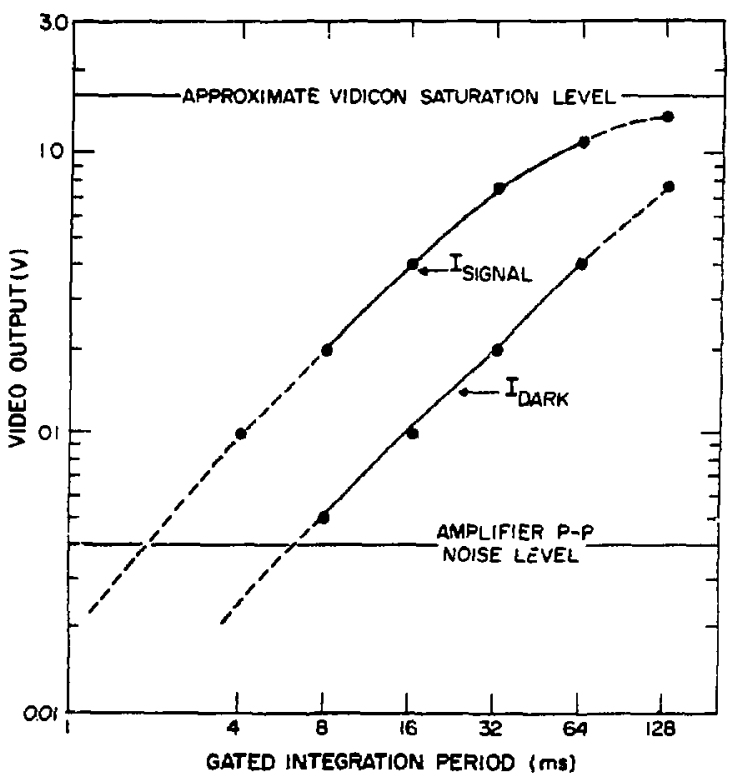

Fig. 27.

Signal and dark-current contributions to the video output signal vs integration time for a Mode'.2 camera with a Z7873 Sb $b_{2} \mathrm{~S}_{\mathrm{a}}$-target vidicon. The dotted lines are extrapolations. 
finished will be correct only for the horizontal line at which the data are taken. However, the transfer characteristic can be corrected if the soak-response curve is also obtained.

The dynamic range is determined, for any given type of tube, by the combined tube and amplifier noise and by tube saturation. At room temperature and except for very short field times, the dark current is the largest component of the tube noise, even for silicon-target tubes. The dark current increases with target voltage and temparature. It limits the dynamic range by using some of the beam current, which is therefore not entirely available for making signals. The dark current therefore compresses the total available dynamic range. The preamp noise is larger than the remaining tube noise (which is primarily the shet-noise contribution from the scanning beam). The preamp noise fixes the low-end limit of the compressed transfer characteristic. The upper end of the dynamic range is limited by several forms of saturation, of which the beam-current and target-bias limits are the most prominent.

Figure 28 shows transfer characteristics for $\mathrm{Sb}_{2} \mathbf{S}_{\mathrm{g}}$ and silicon-target tubes as obtained ising the pulsed-light method. Note the nearly unity gamma at low levels, and the superlinear and sublinear

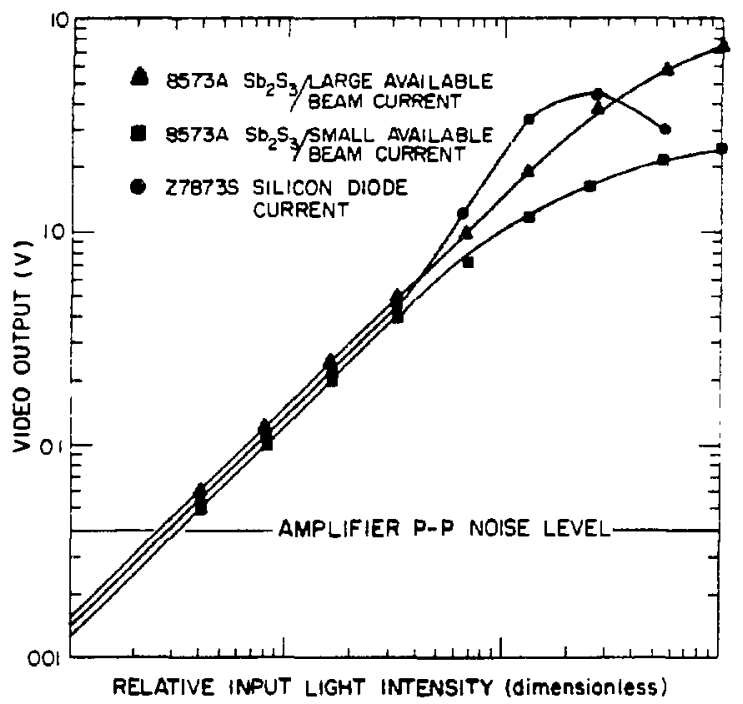

Fig. 28.

Transfer characteristics for $8573 A$ non-FPS $\mathrm{Sb}_{2} \mathrm{~S}_{\mathrm{s}}$-target vidicons with available beam current as a parameter, and $Z 7373 S$ silicon-diodematrix-target FPS vidicon. response at high levels for, respectively, silicon- and $\mathrm{Sb}_{2} \mathrm{~S}_{3}$-target tubes. The $\mathrm{Sb}_{2} \mathrm{~S}_{3}$ curves were obtained with two different total available beam currents. As expected, the larger beam current gives a larger dynamic range and a curve that remains linear to higher light intensities.

\section{B. Contrast-Transfer Function}

We obtain CTF curves by viewing the video amplitude produced by an illuminated bar chart. As for the transfer characteristics, we display one or more horizontal lines on an oscilloscope and note the relative amplitudes of the bars. If desired, the MTF curves are obtained from these data by using a standard conversion formula. ${ }^{*}$ The resolution is ultimately determined by three factors: (1) the inherent resolution of the target and the electron optics; (2) the raster generation rate; and (3), the available overall video-amplifier bandwidih. This last factor was discussed above.

The fundamental grain-size or discrete-devicesize limits discussed in Sec. II. B determine the target resolition jar unic area. The fraction of the target area that is scarned th'ss clearly affects the overall resolution in ternis of ine pairs per picture height. The diameter of the target limits the ultimate resolution. We use only $2.5 \cdot \mathrm{cm}$-diam tubes, which have $18-\mathrm{mm}$-diam targets. The conventional raster dimensions for these targets are $9.5 \mathrm{~mm}$ by $12.7 \mathrm{~mm}$. The size of the rastered portion of the target is especially important if one attempts to increase the scan rate artificially by reducing the raster size. However, the raster size ideally should be only as large as necessary to avoid the fundamental target limit, for two reasons. First, the required sweep voltage is thereby minimizea and, second, the required amplifier bandwidth is also minimized, since lower electronic frequencies are generated for a given scene.

For a given raster size, the raster generation rate does not affect the breakpoint on the CTF curve. This is illustrated in Fig. 29, a plot of video output voltage as a function of spatial frequency for three different horizontal scan rates. The breakpoints are all the same. The slope after the breakpoint is steeper for the $10-\mu \mathrm{s}$ lines because in this case the amplifier bandwidth rolloff is being folded in. Note that this figure also illustrates how the video output increases with the scan rate, as mentioned above. 


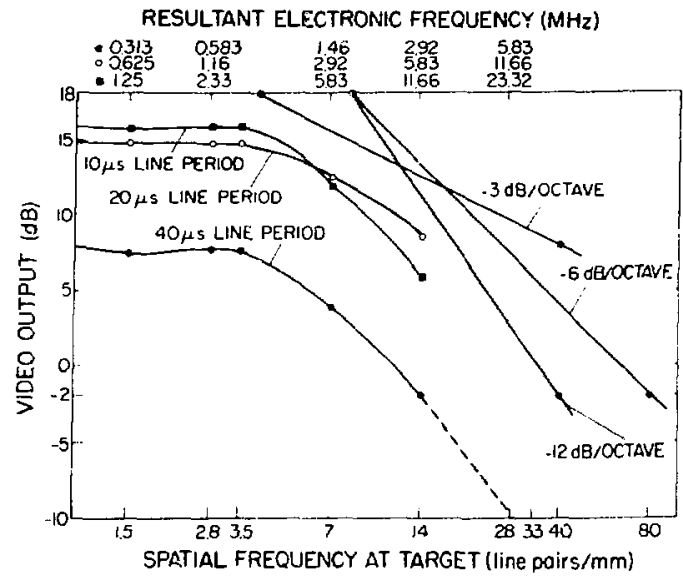

Fig. 29.

CTF curves for three different horizontal scan rates. The data were obtained using a Model-2 camera with a Z7873S silicon-target tube swith a $7-\mathrm{mm}$ by $7-\mathrm{mm}$ raster size.

Figures 30 and 31 show the resolution of a Model-2 camera with an $\mathrm{Sb}_{2} \mathrm{~S}_{3}$ target. Both figuses are monitor photographs of a RETMA resolution chart.
Figure 30 shows the full pattern and Fig. 31 shows a view magnified by increasing only the monitor's raster size so that the resolution wedges can be seen more clearly. The resolution pattern was placed in an Optoliner and flooded with cw light. The raster of appropriate size was generated in the camera using $10-\mu \mathrm{s}$ scan lines. The limiting horizontal resolution is greater than 800 TV lines (TVL). The Optoliner optics and camera raster size $(9.5 \mathrm{~mm}$ by $12.7 \mathrm{~mm}$ at the target) were such that 800 TVL correspond to 32 $\mathrm{LP} / \mathrm{mm}$ at the vidicon target $(800 \mathrm{TVL} / 12.7 \mathrm{~mm}$ times $1 \mathrm{LP} / 2 \mathrm{TVL}$ ). This is about half the limiting resolution quoted by the tube manufacturer for a camera that is scanning at six times the standard rate, that has not been optimized by including phase or aperture corrections, and in which the maximum amplifier bandwidth has been traded for better dynamic range. We conclude that FPS vidicon cameras provide adquate resolution at high scan rates.

We also determined the resolution of this camera using the pulsed-light source and homemade bar chart method discussed at vve. The oscilloscope data are shown in Fig. 32. The sets of bars translate to

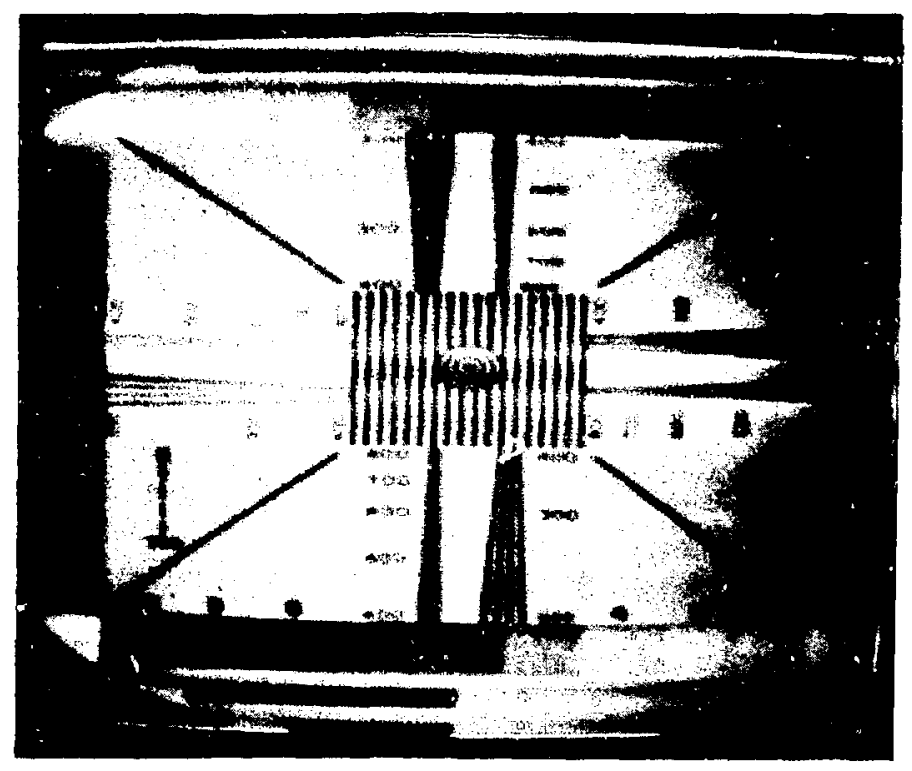

Fig. 30.

Monitor photograph of a standard RETMA resolution chart viewed by a Model-2 camera with an $S b_{n_{3}} \mathbf{S}_{\mathrm{s}}$-target tube using a cw light source. 


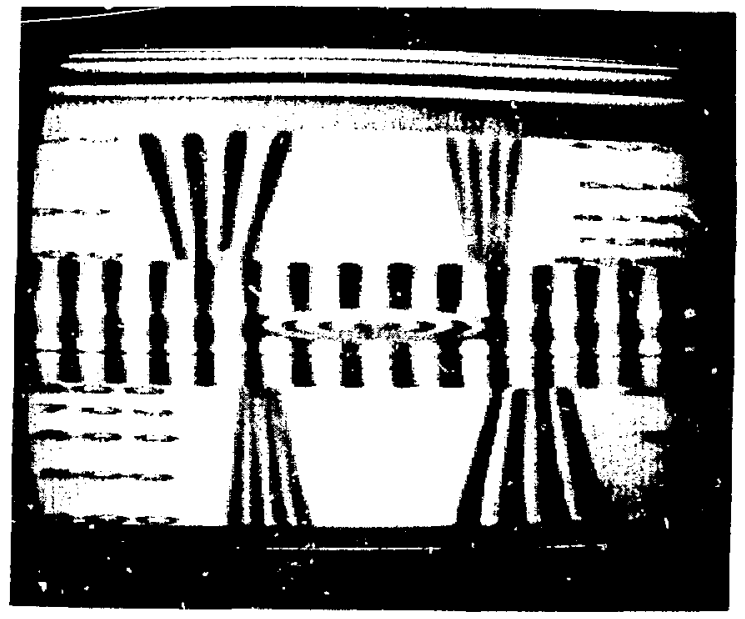

Fig. 31.

Magnified view of a portion of the resolution chart shown in Fig. 30 made by increasing only the monitor's raster size so that the camera parameters are unchanged.

$8.25,16.5$, and $3 \because \mathrm{LP} / \mathrm{mm}$ at the vidicon. The amplitude of the $33 \mathrm{LP} / \mathrm{mm}$ set is $\approx 15 \%$ of the dc or low-frequency response. This result extrapolates to $\approx 40 \mathrm{LP} / \mathrm{mm}$ limiting resolution, which agrees well with the resolution predicted by the nomogram of Fig. 7. We were unable to resolve a $66-\mathrm{LP} / \mathrm{mm}$ set of bars. These results are consistent with the $\mathrm{cw}$ results

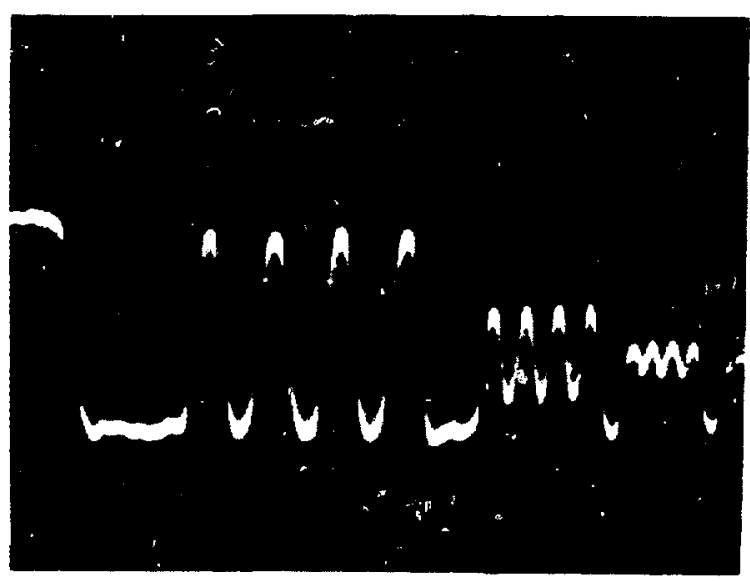

Fig. 32.

Resolution data for a Moc'el-2 camera with a $27873 \mathrm{Sb}_{2} \mathrm{~S}_{8}$-target vidicon and Canon $/ 0.95$ $25-\mathrm{mm}$ lens. One horizontal line is displayed. The horizontal scale is $200 \mathrm{~ns} /$ division; the vertical scale is $200 \mathrm{mV} /$ division. and verify that the camera's resolution does not degrade with $1-\mu \mathrm{S}$-width pulsed light.

We compared the Model-2 camera's resolution with that of our fast-scan (256 $32-\mu$ s scan lines in 8.3 ms) EJSR cameras that use conventional magnetic scanning and focusing. We imaged the same bar chart on both cameras using the pulsed-light method. Both cameras were adjusted for the same out put amplitude, taking into account the different responsivities, video gains, sweep speeds, etc. The crucial parameters, image size on the target and beam speed across the image, were identical for both cameras. Figure 33 shows the resulting waveforms.
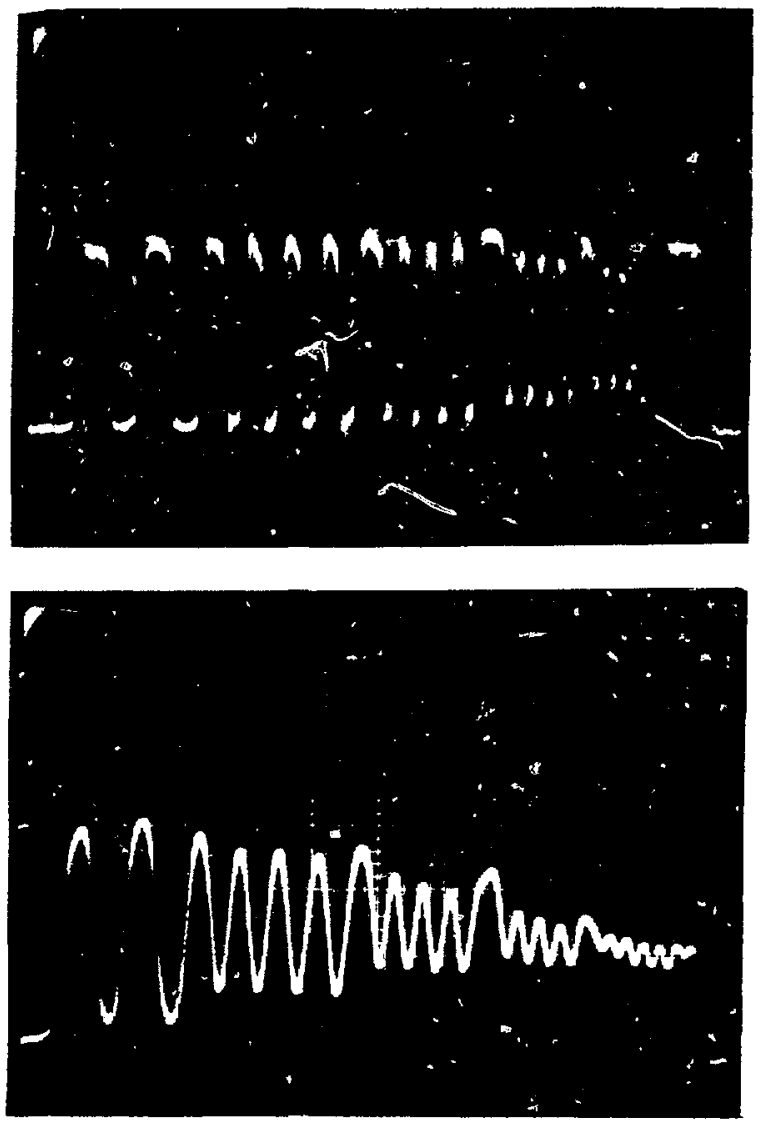

Fig. 33.

Comparision of bar-chart resolution of, at top, an FPS camera and, at bottom, an EDR camera. Waveforms are of one horizontal line viewing a bar-chart pattern. The horizontal scale is $500 \mathrm{~ns} /$ division; the vertical scale is $50 \mathrm{t}$ $\mathrm{mV} /$ division. The sets of bars translate to fundamental electronic frequencies of roughly 2 , $3.5,5,6.5$, and $9 \mathrm{MHz}$. 
The substantial advantage of the Model-2 FPS camera over the EDR camera is readily apparent.

\section{APPLICATIONS}

We will describe briefly several applications in which the Model-1 and -2 FPS cameras have been used.

\section{A. Neutron Pinex,}

The neutron TV pinex (a contraction of pinhole experiment) is a standard diagnostic technique used in underground nuclear tests. The neutrons from a weapon explosion are imaged through a pinhole onto a suitable scintillator, which is viewed by dataacquisition TV cameras placed downhole near the weapon. The cameras record a single field of data during the test. Our FPS cameras were originally designed for use in such experiments and have operated successfully in several tests. They replaced the older EDR cameras. The abilities to select scan rates appropriate to various single-field readout times and to adjust camera parameters remotely are most important in this application. The ability to reset the sync generators with a weapon-triggering signal, the ability to drive long cables to instruments in uphole recording trailers, high video output voltage that helps mask extraneous noise, and good rf shielding are also important.

\section{B. TREAT Experiment}

An adaptation of the neuton TV pinex was used to image fuel motion in fuel pins undergoing planned failure at the Transient Reactor Test (TREAT) Facility at Idaho National Engineering Laboratory. ${ }^{10}$ Cameras tracked the entire transient fuel-pin failure sequence. An FPS camera was used in one of the TREAT experiments. "Two aspects of the TREAT experiments placed extra requirements on the cameras. First, the $\mathrm{S} / \mathrm{N}$ was poor because the fuel pins emitted relatively few neutrons and the background radiation was high. Second, continuous rather than single-field imaging was desired. In the early stages of an experiment, data could be taken relatively slowly because changes in the fuel pin happened slowly. Near the failure time, however, it was desired to take many frames at very rapid rates to gain time resolution.
The camera was fitted with a two-stage image intensifier, which greatly increased sensitivity to the dim images, but at the expense of a large loss of resolution. The manufacturer claims a limiting resolution of $80 \mathrm{LP} / \mathrm{mm}$ at the target for our FPS vidicon. We actually measure $40 \mathrm{LP} / \mathrm{mm}$ for our unintensified cameras. The two-stage intensifier included a proximity-focused diode intensifier and a microchannel-plate intensifier with manufacturerspecified limiting resolutions of 50 and $26 \mathrm{LP} / \mathrm{mm}$, respectively. We measure $13 \mathrm{LP} / \mathrm{mm}$ for the combination. The overall limiting resolution of the camera with the two-stage intensifier was reduced to $<10 \mathrm{LP} / \mathrm{mm}$, or less than one-fourth the unintensified resolution. The camera sensitivity was further increased by adding the integration capability discussed above. The camera's field period was held crnstant at $4 \mathrm{~ms}$, while the integration period between successive field readouts was varied among $4,8,16$, and $32 \mathrm{~ms}$. In this way, the integrated signal amplitude was kept approximately constant even though the incident power varied by a factor of 15 during the transient.

The latch lines of the remotely controllable analog sweep generator (lines $3 \mathrm{D}$ and $4 \mathrm{D}$ in Fig. 12) were driven by the TREAT PDP-11/34 computer, which was programmed to change bit patterns at strategic reactor power levels during the transient power pulse. The $1 \mathrm{D}$ and $2 \mathrm{D}$ latch lines were statically loaded to provide the 4-ms field period. Figure 34 shows the camera's duty cycle during a power pulse. The upper half shows the power and energy in the power pulse as a function of time. The crosses indicate the power levels and the corresponding times at which the camera integration period was changed. The various integration periods are indicated on the second scale from the bottom. For the record, the corresponding bit 0 status, bit 1 status, and CPU strobe pulses are also shown.

Figure 35 illustrates the camera output during four stages of the transient. The peak video output amplitude above background should be proportional to the energy emitted during the integration period. Pertinent data are shown in Table II. The ratio of amplitude to energy is fairly constant, especially considering the low $\mathrm{S} / \mathrm{N}$ and the obvious variation in brightness along the fuel pin. Only in Fig. 35c, representing the highest average power and the largest power variation with time, do the data track poorly. 


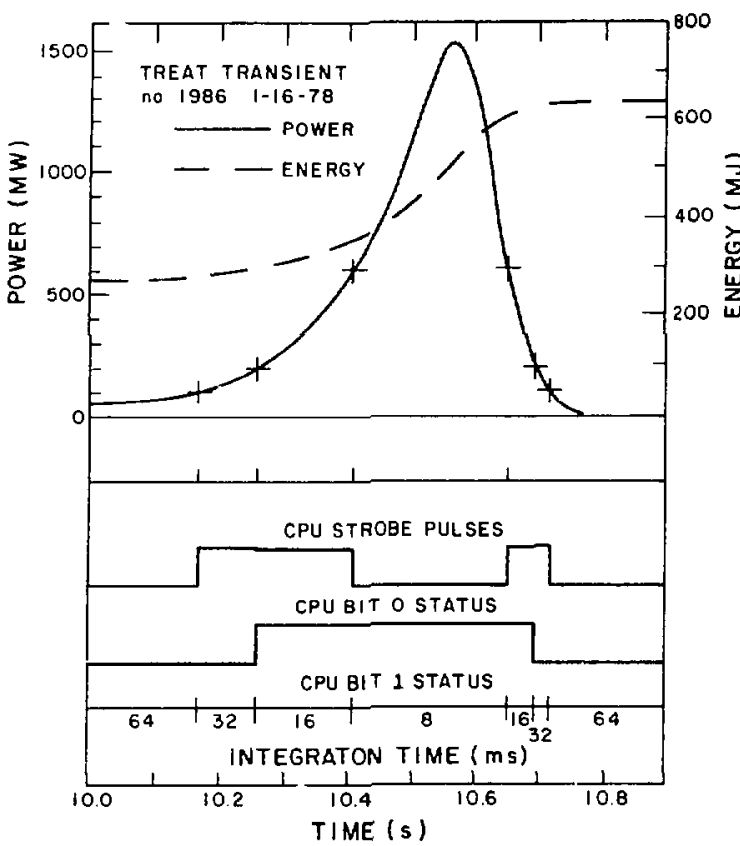

Fig. 34.

TREAT power pulse and corresponding Model-

2 camera integration-time parameters.

The picture quality could be improved by some evolutionary changes in the cameras, by better recording techniques, ${ }^{*}$ and by improving the physical and optical parameters of the experiment to increase the $\mathrm{S} / \mathrm{N}$ to the point that the resolutiondegrading two-stage intensifier could be eliminated.

*At present, the FPS camera's maximum data-acquisition ability greatly exceeds our modest ability to record it.

Fig. 35.

Data from Model-2 camera viewing TREAT fuel pin during power pulse shown in Fig. 34. At left are monitor photographs of the fuel pin at different times during the transient. At right are corresponding digitized oscilloscope waveforms, all adjusted to the same arbitrary scale, of a horizontal line through the data. (a) 32-ms integration prior to pulse peak; (b) 16ms integration prior to pulse peak; (c) 8-ms integration prior to pulse peak; (d) 32-ms integration after pulse peak.
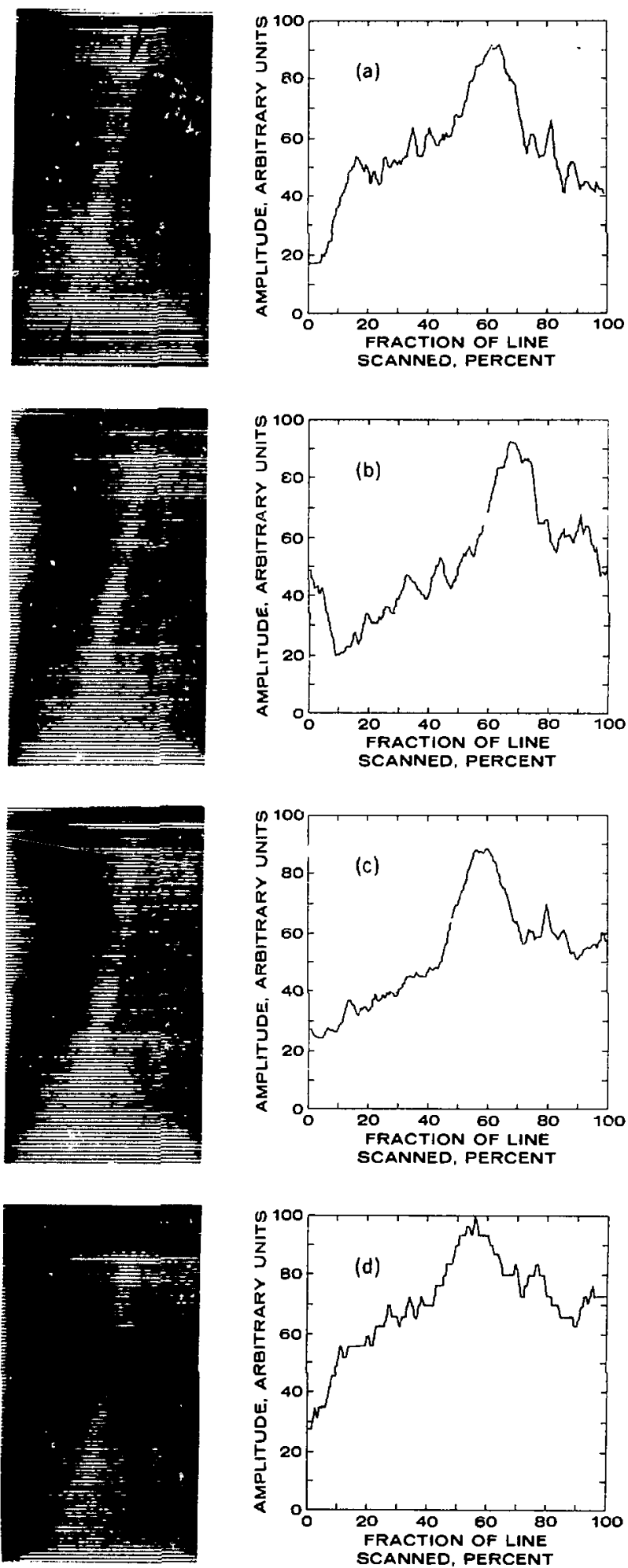
TABLE II

FPS CAMERA PERFORMANCE DURING TREAT TRANSIENT

\begin{tabular}{|c|c|c|c|c|c|}
\hline Figure & $\begin{array}{c}\text { Integration } \\
\text { Period } \\
\text { (ms) }\end{array}$ & $\begin{array}{c}\text { Average } \\
\text { Power } \\
\text { (MW) }\end{array}$ & $\begin{array}{c}\text { Energy } \\
\text { (MJ) }\end{array}$ & $\begin{array}{c}\text { Video } \\
\text { Output } \\
\text { Amplitude }\end{array}$ & $\begin{array}{l}\text { Ratio of } \\
\text { Amplitude } \\
\text { to Energy }\end{array}$ \\
\hline $35 \mathrm{a}$ & 32 & 175 & 5.6 & 28 & 5.0 \\
\hline $35 b$ & 16 & 350 & 5.6 & 34 & 6.1 \\
\hline $35 \mathrm{c}$ & 8 & 1070 & 8.6 & 36 & 4.2 \\
\hline $3 \bar{j} \mathrm{~d}$ & 32 & 150 & 4.8 & 23 & 4.8 \\
\hline
\end{tabular}

\section{Barium-Release Experiment}

Certain studies of the earth's upper atmosphere involve releasing rocket-borne shaped charges of barium. The barium is released at an altitude that is in sunlight while the ground station is in darkness. The barium ionizes by shock and photoionization. The resultant plasma cloud forms into patierns caused by the earth's geomagnetic field. The ions luminesce by resonant scattering in sunlight. Viewing the luminescence provides a tool to study various physical effects. In an experiment called Avefria, the barium was released into the ionosphere at a $195 \cdot \mathrm{km}$ altitude to study striations caused by the shaped charge's injecting plasma across the geomagnetic field and to study communications occlusions through a striated plasma. For the latter study, the striations were formed between an earth-orbiting communications satellite and a receiver at the ground station.

The cainera used to study the striations was a high-resolution large-diameter-target image orthicon with a narrow field of view. A more sensitive but lower-resolution doubly intensified FPS camera with a wide field of view was used to locate the cloud initially. Figure 36 is a composite photogranh of the bariurn cloud at a sequence of

limes during the early part of its growth. A known star pattern in this part of the night sky was used belore the event to locate approximately the cloud's 'xpected position with respect to the known position "If the matlite. Because the event time occurred just belore clawn, it was necessary to attenuate the strong batkground from the lightening sky by fitting the (amera lens with a narrowband $455.4-\mathrm{nm}$ inffrlerence tilter that passed only the light from the ('lud, 'The stars are therefore not visible in Fig. 36 . The intensity of the cloud varied from $2 \times 10^{-12}$ to a maximum of $2 \times 10^{-4} \mathrm{~W} / \mathrm{cm}^{2} \cdot \mathrm{sr}$. The resolution of the (wo)-stage intensifier sufficiently degraded the werali resolution so that the FPS camera was unable w clearly see the striations that formed later.

\section{ACKNOWLEDGMENTS}

'The authors wish to thank the large number of perple who assisted in the specification, design, tabrication, testing, and applications phases of these cameras and those who provided helpful suggestions and discussions. 'They include J. Gai ;ia, E. Ferron, J. H. Calligan III, G, Barber, V. H. Holmes, Jr., K. Gintierrez, M. Cates, G. Smith, W. M. Sanders, D. Phillips, R. Partridge, F. Honey, R. Hiebert, D.

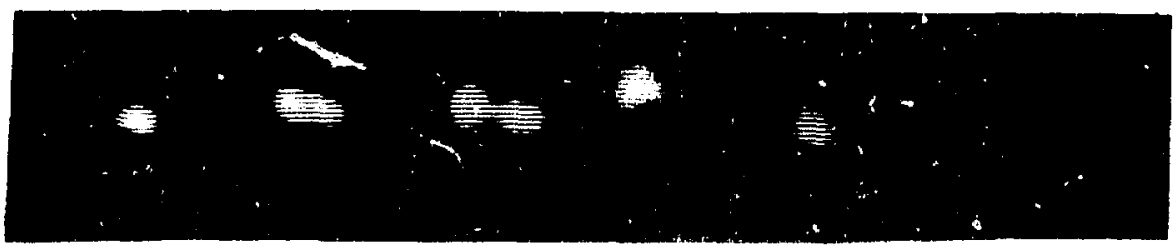

Fig. 36 .

Composite photograph of barium cloud. 
Brown, J. Lunsford, G. Berzins, and J. Wolcottt of Los Alamos Scientific Laboratory; D. Mannikko, E. Wilson. G. Simon, A. Kvanvig, and D. LiButti of EG\&G Inc. Energy Measurements group; D. Dunbar of Lawrence Livermore Laboratory; and R. Wagner, J. Fischer and D. Hunter of General Electric Co., Imaging Devices Operation.

\section{REFERENCES}

1. K. Schlesinger and R. A. Wagner, "A MixedField Device for Simultaneous Focus and Deflection," Proc. Electron and Laser Beam Symp., Pernsylvania State University, University Park, Pennsylvania, March 31-April 2, 1965 (Electrical Enginfering Department, Electron Physics Laboratory, Pennsylvania State University, University Park, PA 16802, 1965) pp. 471488 .

2. K. Schlesinger and R. A. Wagner, "A Mixed Field Type of Vidicon," IEEE Trans. Electron Devices ED-14, 163-170 (1967).

3. I. T. Saldi and K. Schlesinger, "The FPS Vidicon," Opt. Spectra 4, 53-56 (February 1970).

4. D. B. Hunter, "Supertube," Opt. Spectra 9, 3941 (November 1975).
5. K. Schlesinger, "Progress in the Development of Post-Acceleration and Electrostatic Deflection," Proc. IRE 44, 659-667 (1956).

6. G. J. Yates and B. W. Noel, "A 256-line, 2.8-ms Field Duration TV Camera," Los Alamos Scientific Laboratory report LA-6407 (November 1976).

7. G. J. Yates, "Fast Scan TV System, "Los Alamos Scientific Laboratory, unpublished report, 1972.

8. G. J. Yates and V. H. Holmes, Jr., "Typical Vidicon Responses to Short-Duration Pulsed Light and Fast Single-Field Readout," Los Alamos Scientific Laboratory report LA-7026 (March 1978).

9. RCA Electro-Optics Handbook (RCA, Commercial Engineering, Harrison, New Jersey, 1974), pp.114-117.

10. G. J. Berzins and K. S. Han, "Pinhole Imaging of a Test Fuel Element at the Transient Reactor Test Facility," Nucl. Sci. Eng. 65, 28-40 (1978).

11. G. J. Berzins and A. H. Lumpkin, "Pinex-2: Pinhole-TV Imaging of Fuel Ejection from an Internally Vented Capsule," Proc. Ir.t. Meeting on Fast Reactor Safety Technology, Seattle, Washington, Aligust 19-23, 1979 (to be published). 